\title{
Exceptional Laguerre Polynomials
}

\author{
By Niels Bonneux and Arno B.J. Kuijlaars
}

The aim of this paper is to present the construction of exceptional Laguerre polynomials in a systematic way and to provide new asymptotic results on the location of the zeros. To describe the exceptional Laguerre polynomials, we associate them with two partitions. We find that the use of partitions is an elegant way to express these polynomials and we restate some of their known properties in terms of partitions. We discuss the asymptotic behavior of the regular zeros and the exceptional zeros of exceptional Laguerre polynomials as the degree tends to infinity.

\section{Introduction}

Laguerre polynomials are one of the three classes of classical orthogonal polynomials [1], next to Hermite and Jacobi polynomials. These classical orthogonal polynomials are very well understood. In the past few years, Laguerre polynomials are extended to exceptional Laguerre polynomials [2-10], also sometimes called multi-indexed Laguerre polynomials [11-16]. The remarkable new feature of this generalization is that the exceptional Laguerre polynomial does not exist for every degree, as was first discovered by Gómez-Ullate et al. [3]. Because of this unusual property, these polynomials were called exceptional. Remarkably, the exceptional Laguerre polynomials may still form a complete orthogonal system [2,17]. The

Address for correspondence: N. Bonneux and A. B. J. Kuijlaars, Department of Mathematics, Katholieke Universiteit Leuven, Celestijnenlaan 200B, Box 2400, 3001 Leuven, Belgium; e-mail: niels.bonneux@kuleuven.be and arno.kuijlaars@kuleuven.be 
orthogonality condition only holds for the admissible degrees and they are complete in their natural Hilbert space setting.

Besides exceptional Laguerre polynomials, there are also exceptional Hermite and exceptional Jacobi polynomials. Recently, García-Ferrero et al. classified exceptional orthogonal polynomials [18] and it turns out that every system of exceptional orthogonal polynomials is related to one of the classical orthogonal polynomials by a sequence of Darboux transformations $[19,20]$. Hence, there are only three families: exceptional Hermite, exceptional Laguerre, and exceptional Jacobi polynomials. These exceptional polynomial systems appear in quantum mechanical problems as solutions in exactly solvable models [21,22] or in superintegrable systems [23].

From a mathematical point of view, there are two main questions. First, there is the question about the classification of these polynomials. This issue is basically solved in [18]. Second, there is the question about the properties of the exceptional orthogonal polynomials. In particular, one could wonder whether these polynomials have similar properties as their classical counterparts. A noticeable difference is that orthogonal polynomials only have real zeros, while the exceptional orthogonal polynomials can have nonreal zeros.

The purpose of this paper is twofold. We give an overview of the construction and classification of exceptional Laguerre polynomials using the concept of partitions. Next, we derive asymptotic results about the zeros of these polynomials. In many cases, the exceptional Laguerre polynomials are a complete set of eigenpolynomials in an appropriate Hilbert space where boundary conditions have to be taken into account. Although this is an important issue, we do not consider it in this paper.

We construct the exceptional Laguerre polynomial via two partitions [24] as we feel that this is the most natural setting for studying these polynomials. Such a construction by partitions is already used for exceptional Hermite polynomials, see, for example $[25,26]$. In the Laguerre case, we show how a pair of partitions can be used to construct exceptional Laguerre polynomials and we give some of their properties. The properties are not new as they can be found in various places in the literature with varying notation. We find it useful to present it here in a systematic way. In some of the existing literature, the exceptional Laguerre polynomials are classified as type I, type II, and type III $X_{m}$-Laguerre polynomials, see, e.g. [7,27]. This classification captures only very specific cases for the exceptional Laguerre polynomial. We show how to rewrite the $X_{m}$-Laguerre polynomials in our approach using partitions, see Section 5.2.

The second aim of this paper is to address the question about the asymptotic behavior of the zeros of the exceptional Laguerre polynomial. This asymptotic behavior is studied in the Hermite case [26] where the corresponding partition is chosen to be even. We use similar ideas in this 
paper to obtain the new results for the Laguerre case. Our results partially answer the conjecture made in [26] that their result in the Hermite case should hold in the Laguerre and Jacobi case as well. The behavior of the zeros of some exceptional Laguerre polynomials is already studied in the papers [27-29]. In these papers, only 1-step Darboux transformations are considered whereas we deal with general $r$-step transformations, thereby covering all possible cases.

The zeros are divided into two classes according to their location. The zeros which lie in the orthogonality region are called the regular zeros, while the others are called the exceptional zeros. Our first result is a lower bound for the number of regular zeros. Next, we prove a generalization of the Mehler-Heine theorem of Laguerre polynomials. We also show that the counting measure of the regular zeros, suitably normalized, converges to the Marchenko-Pastur distribution. This distribution is also the limiting distribution of the scaled zeros of classical Laguerre polynomials. Finally, we prove that each simple zero of the generalized Laguerre polynomial attracts an exceptional zero of the exceptional Laguerre polynomial. The condition that we need simple zeros is probably not a restriction. In the Hermite case, it was conjectured by Veselov [30] that the zeros of a Wronskian of a fixed set of Hermite polynomials are indeed simple, except possibly at the origin. Likewise, we conjecture that the zeros of the generalized Laguerre polynomial are simple too. An important difference with Veselov's conjecture is that we add a condition in our statement. The zeros are not simple in full generality as we have explicit examples where nonsimple zeros are obtained.

We organize this paper as follows. In Section 2, we introduce the generalized and exceptional Laguerre polynomial by associating them with two partitions. After defining them properly, we give an alternative proof to derive the degree and leading coefficient of these polynomials in Section 3. In Section 4, we go on to discuss the most general construction of generalized Laguerre polynomials. The most general construction involves four types of eigenfunctions of the Laguerre differential operator, which could be captured by four partitions. However, there is a procedure with Maya diagrams to reduce it to only two types. We follow [31] who worked out the reduction procedure for the Hermite case. The construction of the exceptional Laguerre polynomials in the most general case is treated in Section 5. In this section, we also give the relation between our approach using partitions and $X_{m}$-Laguerre polynomials.

Finally, we state and prove the new results about the zeros of the exceptional Laguerre polynomial in 6 and 7. These sections are divided into the results according to the regular and exceptional zeros. We also state a conjecture of simple zeros in 6 and give some remarks about it. 


\section{Table 1}

Eigenfunctions and Eigenvalues of (2)

\begin{tabular}{lc}
\hline Eigenfunction & Eigenvalue \\
\hline$L_{n}^{(\alpha)}(x)$ & $-n$ \\
$e^{x} L_{n}^{(\alpha)}(-x)$ & $n+1+\alpha$ \\
$x^{-\alpha} L_{n}^{(-\alpha)}(x)$ & $-n+\alpha$ \\
$x^{-\alpha} e^{x} L_{n}^{(-\alpha)}(-x)$ & $n+1$ \\
\hline
\end{tabular}

\section{Exceptional Laguerre polynomials}

We start from Laguerre polynomials to define generalized and exceptional Laguerre polynomials where we introduce them by use of partitions. After the necessary definitions, we state a few known results for these polynomials and give an elementary duality property for the partitions. Most of the results of this section can be found in $[2,32]$.

\subsection{Laguerre polynomials}

The Laguerre polynomial of degree $n$ with parameter $\alpha \in \mathbb{R}$ is given by the Rodrigues formula

$$
L_{n}^{(\alpha)}(x)=\frac{e^{x} x^{-\alpha}}{n !} \frac{d^{n}}{d x^{n}}\left(e^{-x} x^{n+\alpha}\right) .
$$

For $\alpha>-1$, they are orthogonal polynomials on $[0, \infty)$ with respect to the positive weight function $x^{\alpha} e^{-x}$,

$$
\int_{0}^{\infty} L_{n}^{(\alpha)}(x) L_{m}^{(\alpha)}(x) x^{\alpha} e^{-x} d x=0, \quad \text { for } n \neq m .
$$

The Laguerre polynomial $L_{n}^{(\alpha)}$ is an eigenfunction of the differential operator

$$
y \mapsto x y^{\prime \prime}+(\alpha+1-x) y^{\prime}
$$

There are other eigenfunctions of this operator which have a polynomial part and these are listed in Table 1. We will use these eigenfunctions to define the generalized and exceptional Laguerre polynomial. As we see later, the first two types of eigenfunctions are the most relevant ones for us.

We can transform the operator (2) into

$$
y \mapsto-y^{\prime \prime}+\left(x^{2}+\frac{4 \alpha^{2}-1}{4 x^{2}}\right) y,
$$


Table 2

Eigenfunctions and Eigenvalues of (3)

\begin{tabular}{lc}
\hline Eigenfunction & Eigenvalue \\
\hline$\varphi_{n}^{(\alpha)}(x)=e^{-\frac{1}{2} x^{2}} x^{\alpha+\frac{1}{2}} L_{n}^{(\alpha)}\left(x^{2}\right)$ & $4 n+2(1+\alpha)$ \\
$\psi_{n}^{(\alpha)}(x)=e^{\frac{1}{2} x^{2}} x^{\alpha+\frac{1}{2}} L_{n}^{(\alpha)}\left(-x^{2}\right)$ & $-4 n-2(1+\alpha)$ \\
$\varphi_{n}^{(-\alpha)}(x)=e^{-\frac{1}{2} x^{2}} x^{-\alpha+\frac{1}{2}} L_{n}^{(-\alpha)}\left(x^{2}\right)$ & $4 n+2(1-\alpha)$ \\
$\psi_{n}^{(-\alpha)}(x)=e^{\frac{1}{2} x^{2}} x^{-\alpha+\frac{1}{2}} L_{n}^{(-\alpha)}\left(-x^{2}\right)$ & $-4 n-2(1-\alpha)$ \\
\hline
\end{tabular}

in the sense that if $y$ is an eigenfunction of (2), then $x^{\alpha+\frac{1}{2}} e^{-\frac{1}{2} x^{2}} y\left(x^{2}\right)$ is an eigenfunction of (3). The corresponding eigenfunctions are given in Table 2 and we denote them by $\varphi_{n}^{(\alpha)}$ and $\psi_{n}^{(\alpha)}$. The Laguerre polynomial $L_{n}^{(\alpha)}$ itself transforms into

$$
\varphi_{n}^{(\alpha)}(x)=x^{\alpha+\frac{1}{2}} e^{-\frac{1}{2} x^{2}} L_{n}^{(\alpha)}\left(x^{2}\right)
$$

From $\varphi_{n}^{(\alpha)}$, we obtain the eigenfunction $\psi_{n}^{(\alpha)}$ of (3) by changing $x$ to $i x$ in (4). Two more eigenfunctions are obtained by changing $\alpha$ to $-\alpha$. We arrive at four sets of eigenfunctions with a polynomial part as shown in Table 2 . The operator (4) is in the Schrödinger form and it is convenient to apply a Darboux-Crum (or a sequence of Darboux) transformation(s) to this form of the operator $[19,20]$.

\subsection{Generalized Laguerre polynomials}

We will now define the generalized Laguerre polynomial. Let us, however, point out that the term generalized Laguerre polynomial is also used for the polynomial (1) with general parameter $\alpha$, to distinguish it from the case $\alpha=0$ (which is then called the Laguerre polynomial). We use generalized Laguerre polynomial in analogy with generalized Hermite polynomial as for example in [26].

For us, the generalized Laguerre polynomial is basically a Wronskian of the eigenfunctions in Table 1 with an appropriate prefactor to make it a polynomial. A Wronskian of a set of sufficiently differentiable functions $f_{1}, \ldots, f_{r}$ is the determinant of the matrix $\left(\frac{d^{j-1}}{d x^{j-1}} f_{i}\right)_{1 \leq i, j \leq r}$. We denote it by

$$
\mathrm{Wr}\left[f_{1}, \ldots, f_{r}\right]=\operatorname{det}\left(\frac{d^{j-1}}{d x^{j-1}} f_{i}\right)_{1 \leq i, j \leq r} .
$$

A generalized Laguerre polynomial is a Wronskian of $r$ Laguerre polynomials with the same parameter $\alpha$, but with distinct degrees $n_{1}>n_{2}>\cdots>$ $n_{r} \geq 1$. We label the generalized Laguerre polynomial by the partition 
$\lambda=\left(\lambda_{1} \geq \cdots \geq \lambda_{r}\right)$, where $\lambda_{j}=n_{j}-r+j$ for $j=1, \ldots, r$. Then,

$$
\Omega_{\lambda}^{(\alpha)}:=\operatorname{Wr}\left[L_{n_{1}}^{(\alpha)}, \ldots, L_{n_{r}}^{(\alpha)}\right]
$$

is a polynomial of degree $|\lambda|=\sum_{j=1}^{r} \lambda_{j}$. The fact that $|\lambda|$ is the highest degree can be easily seen by looking at the diagonal or antidiagonal. The coefficient to $x^{|\lambda|}$ is nonzero because all the positive integers $n_{j}$ are different. See Lemma 1 for a precise formula of the leading coefficient. Note that we follow the convention that a partition is a weakly decreasing sequence of positive integers, which is the common one in number theory and combinatorics.

A more general approach is possible if we include other eigenfunctions of the operator (2). Because we want to end up with polynomials, we only include the eigenfunctions from Table 1. In general, we could include all four types, but it turns out that it suffices to include only eigenfunctions of the first two types, see Section 4. We consider two partitions $\lambda$ and $\mu$ of lengths $r_{1}$ and $r_{2}$ with corresponding degree sequences $\left(n_{j}\right)_{j=1}^{r_{1}}$ and $\left(m_{j}\right)_{j=1}^{r_{2}}$,

$$
\begin{array}{lll}
\lambda=\left(\lambda_{1}, \ldots, \lambda_{r_{1}}\right), & n_{j}=\lambda_{j}+r_{1}-j, & j=1, \ldots, r_{1}, \\
\mu=\left(\mu_{1}, \ldots, \mu_{r_{2}}\right), & m_{j}=\mu_{j}+r_{2}-j, & j=1, \ldots, r_{2} .
\end{array}
$$

We write $r=r_{1}+r_{2}$ and

$$
\Omega_{\lambda, \mu}^{(\alpha)}:=e^{-r_{2} x} \cdot \operatorname{Wr}\left[f_{1}, \ldots, f_{r}\right],
$$

where

$$
\begin{array}{cc}
f_{j}(x)=L_{n_{j}}^{(\alpha)}(x), & j=1, \ldots, r_{1}, \\
f_{r_{1}+j}(x)=e^{x} L_{m_{j}}^{(\alpha)}(-x), & j=1, \ldots, r_{2} .
\end{array}
$$

The prefactor $e^{-r_{2} x}$ in (7) guarantees that $\Omega_{\lambda, \mu}^{(\alpha)}$ is a polynomial. We see that $\Omega_{\lambda, \emptyset}^{(\alpha)}=\Omega_{\lambda}^{(\alpha)}$.

Definition 1. The polynomial $\Omega_{\lambda, \mu}^{(\alpha)}$ defined in (7) is called the generalized Laguerre polynomial of parameter $\alpha$ associated with partitions $\lambda$ and $\mu$.

When both partitions are empty, the generalized Laguerre polynomial is the constant function 1.

Remark 1. As the Laguerre polynomial, the generalized Laguerre polynomial is defined for every $\alpha \in \mathbb{R}$ and the polynomial never vanishes identically, because the functions $f_{1}, \ldots, f_{r}$ from (8) to (9) are always linearly independent. As we can see from Table 1, combined with our definition of $\Omega_{\lambda, \mu}^{(\alpha)}$, it is possible that for a particular choice of $\alpha$, namely, 
$\alpha=-n_{i}-m_{j}-1$ for some $i$ and $j$, two eigenvalues coincide. Nevertheless, the eigenfunctions are still linearly independent as one of them has an exponential part, while the other one has not.

If we apply a few elementary properties of Laguerre polynomials (which are stated further, see (48) and (49)), we can express the generalized Laguerre polynomial (7) as

$$
\left|\begin{array}{cccccc}
L_{n_{1}}^{(\alpha)}(x) & \ldots & L_{n_{r_{1}}}^{(\alpha)}(x) & L_{m_{1}}^{(\alpha)}(-x) & \ldots & L_{m_{r_{2}}}^{(\alpha)}(-x) \\
(-1) L_{n_{1}-1}^{(\alpha+1)}(x) & \ldots & (-1) L_{n_{r_{1}}-1}^{(\alpha+1)}(x) & L_{m_{1}}^{(\alpha+1)}(-x) & \ldots & L_{m_{r_{2}}}^{(\alpha+1)}(-x) \\
\vdots & \ddots & \vdots & \vdots & \ddots & \vdots \\
(-1)^{r-1} L_{n_{1}-r+1}^{(\alpha+r-1)}(x) & \ldots & (-1)^{r-1} L_{n_{r_{1}}-r+1}^{(\alpha+r-1)}(x) & L_{m_{1}}^{(\alpha+r-1)}(-x) & \ldots & L_{m_{r_{2}}}^{(\alpha+r-1)}(-x)
\end{array}\right| \text {, }
$$

where we set $L_{n}^{(\alpha)}(x) \equiv 0$ whenever $n \leq-1$. The expression (10) shows that our definition of $\Omega_{\lambda, \mu}^{(\alpha)}$ is the same as Durán's definition [2, Formula (1.8)]. Using this expression, one can easily see that the degree of $\Omega_{\lambda, \mu}^{(\alpha)}$ is at most $|\lambda|+\sum_{i=1}^{r_{2}} m_{i}$. However, in this situation, there is a fair amount of cancellation and we have the following result from Durán [2, Section 5].

Lemma 1. Take $\alpha \in \mathbb{R}$. Then, $\Omega_{\lambda, \mu}^{(\alpha)}$ is a polynomial of degree $|\lambda|+|\mu|$ with leading coefficient given by

$$
(-1)^{\sum_{i=1}^{r_{1}} n_{i}} \cdot \frac{\prod_{1 \leq i<j \leq r_{1}}\left(n_{j}-n_{i}\right) \prod_{1 \leq i<j \leq r_{2}}\left(m_{j}-m_{i}\right)}{\prod_{i=1}^{r_{1}} n_{i} ! \prod_{i=1}^{r_{2}} m_{i} !},
$$

which is independent of the parameter $\alpha$.

Actually, Durán [2] first considers exceptional Meixner polynomials and obtains Wronskians of Laguerre polynomials as limits of Casorati determinants of Meixner polynomials. The degree statement for these latter determinants follows from a quite general result of Durán and de la Iglesia [33, Lemma 3.4]. In Section 3, we give a direct argument of the degree reduction. Therefore, Lemma 1 is the Laguerre case of Proposition 1 where we use that the leading coefficient of the Laguerre polynomial $L_{n}^{(\alpha)}(x)$ is given by $\frac{(-1)^{n}}{n !}$.

The value at the origin of the generalized Laguerre polynomial $\Omega_{\lambda, \mu}^{(\alpha)}$ can be computed explicitly. Durán showed that this value is nonzero when the parameter $\alpha$ is not a negative integer [2, Lemma 5.1]. By investigating this lemma of Durán, we easily verify that the condition can be weakened.

Lemma 2. Take $\alpha \in \mathbb{R}$ such that the following conditions are satisfied,

$$
\alpha \neq-1,-2, \ldots,-\max \left\{n_{1}, m_{1}\right\},
$$




$$
\alpha \neq-n_{i}-m_{j}-1, \quad i=1, \ldots, r_{1} \text { and } j=1, \ldots, r_{2} .
$$

Then, $\Omega_{\lambda, \mu}^{(\alpha)}(0) \neq 0$.

The statement of Lemma 2 is not best possible, but an explicit formulation of the values that are not allowed for $\alpha$ is rather complicated to write down, and we not pursue this in this paper. The condition $\Omega_{\lambda, \mu}^{(\alpha)}(0) \neq 0$ will play a role in Corollary 1 in Section 6.2.

The two partitions play a similar role in (6) as is evident from the following duality property.

LeMma 3. For every $\alpha \in \mathbb{R}$ and partitions $\lambda$ and $\mu$, we have

$$
\Omega_{\lambda, \mu}^{(\alpha)}(x)=(-1)^{\frac{r_{1}\left(r_{1}-1\right)}{2}+\frac{r_{2}\left(r_{2}-1\right)}{2}} \Omega_{\mu, \lambda}^{(\alpha)}(-x) .
$$

Proof. We use the following elementary Wronskian properties. Assume that $f_{1}, \ldots, f_{r}, g$, and $h$ are sufficiently differentiable. Then,

$$
\begin{gathered}
\operatorname{Wr}\left[g \cdot f_{1}, \ldots, g \cdot f_{r}\right]=(g(x))^{r} \cdot \operatorname{Wr}\left[f_{1}, \ldots, f_{r}\right], \\
\operatorname{Wr}\left[f_{1} \circ h, \ldots, f_{r} \circ h\right](x)=\left(h^{\prime}(x)\right)^{\frac{r(r-1)}{2}} \cdot \operatorname{Wr}\left[f_{1}, \ldots, f_{r}\right](h(x)) .
\end{gathered}
$$

Then, from (7) and (12) with $g(x)=e^{-x}$, we have

$$
\Omega_{\lambda, \mu}^{(\alpha)}=e^{r_{1} x} \cdot \operatorname{Wr}\left[e^{-x} f_{1}, \ldots, e^{-x} f_{r}\right],
$$

where $f_{1}, \ldots, f_{r}$ are the functions from (8) to (9). By using (13) with $h(x)=-x$, we obtain

$$
\Omega_{\lambda, \mu}^{(\alpha)}(x)=(-1)^{\frac{r(r-1)}{2}} e^{r_{1} x} \cdot \operatorname{Wr}\left[g_{r_{2}+1}, \ldots, g_{r}, g_{1}, \ldots, g_{r_{2}}\right](-x),
$$

where $g_{j}(x)=e^{x} f_{r_{1}+j}(-x)=L_{m_{j}}^{(\alpha)}(x)$ for $j=1, \ldots, r_{2}$ and $g_{r_{2}+j}(x)=$ $e^{x} f_{j}(-x)=e^{x} L_{n_{j}}^{(\alpha)}(-x)$ for $j=1, \ldots, r_{1}$. Permuting the first $r_{1}$ columns with the last $r_{2}$ columns gives an extra factor $(-1)^{r_{1} r_{2}}$. Therefore, the total factor is

$$
(-1)^{r_{1} r_{2}+\frac{r(r-1)}{2}}=(-1)^{\frac{r_{1}\left(r_{1}-1\right)}{2}+\frac{r_{2}\left(r_{2}-1\right)}{2}} .
$$

Hence, we obtain (11) in view of the definitions (7)-(9).

Durán [2] gave sufficient conditions for $\Omega_{\lambda, \mu}^{(\alpha)}$ to have no zeros on $[0, \infty)$. In a follow-up paper, Durán and Pérez [17] proved that the obtained conditions are also necessary. For their result, we need the notion of an even partition.

Definition 2. A partition $\lambda=\left(\lambda_{1}, \ldots, \lambda_{r}\right)$ with $\lambda_{r} \geq 1$ is even if $r$ is even and $\lambda_{2 j-1}=\lambda_{2 j}$ for every $j=1, \ldots, \frac{r}{2}$.

By convention, when $r=0$, the (empty) partition is even. 


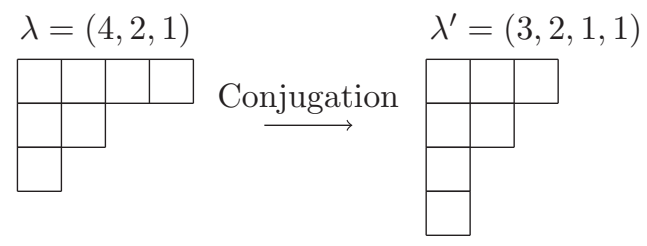

Figure 1. The conjugate partition.

We state the following result for $\alpha>-1$, even though Durán and Pérez obtain a result for every $\alpha \in \mathbb{R}$.

LEMMA 4 ([2,17]). Let $\alpha>-1$. Then the polynomial $\Omega_{\lambda, \mu}^{(\alpha)}$ has no zeros on $[0, \infty)$ if and only if $\lambda$ is an even partition.

Suppose $\alpha>-1$. Combining Lemmas 4 and 3 gives us that $\Omega_{\lambda, \mu}^{(\alpha)}$ has no zeros on $(-\infty, 0]$ if and only if $\mu$ is an even partition. Moreover, $\Omega_{\lambda, \mu}^{(\alpha)}$ has no real zeros if and only if both $\lambda$ and $\mu$ are even partitions.

Finally, we state an invariance property which was conjectured in [2] and proven in [32]. This property is very conveniently stated in terms of partitions, because it involves the conjugate partition. The partition $\lambda^{\prime}$ is called the conjugate partition of $\lambda$ if

$$
\lambda_{i}^{\prime}=\#\left\{j \in \mathbb{N} \mid \lambda_{j} \geq i\right\}, \quad i=1, \ldots, \lambda_{1} .
$$

If we use a graphical representation of a partition by means of a Young diagram, then the Young diagram of the conjugate partition is obtained by reflection in the main diagonal, as illustrated in Fig. 1.

LEMma 5 (Theorem 6.1 in [32]). For every $\alpha \in \mathbb{R}$ and partitions $\lambda$ and $\mu$, we have

$$
\Omega_{\lambda, \mu}^{(\alpha)}(x)=(-1)^{\frac{\mu_{1}\left(\mu_{1}-1\right)}{2}+|\lambda|+|\mu|+\frac{r_{2}\left(r_{2}-1\right)}{2}} \Omega_{\lambda^{\prime}, \mu^{\prime}}^{(-\alpha-t)}(-x),
$$

where

$$
t=\lambda_{1}+\mu_{1}+r_{1}+r_{2}
$$

with the convention that $\lambda_{1}=0$ if $r_{1}=0$ (i.e., $\lambda$ is the empty partition) and $\mu_{1}=0$ if $r_{2}=0$.

Lemma 5 will follow from Theorem 1 in Section 4. The proof is given in Section 4.5 .

\subsection{Exceptional Laguerre polynomials}

We fix the parameter $\alpha$ and two partitions $\lambda$ and $\mu$ of lengths $r_{1}$ and $r_{2}$, respectively. As before, we write $r:=r_{1}+r_{2}$. Furthermore, suppose that the functions $f_{1}, \ldots, f_{r}$ are as in (8) and (9). We obtain the exceptional 
Laguerre polynomials by adding one Laguerre polynomial with the same parameter $\alpha$ but with degree different from $n_{1}, \ldots, n_{r_{1}}$ to the Wronskian. If we add the Laguerre polynomial of degree $s$, then this is

$$
e^{-r_{2} x} \cdot \operatorname{Wr}\left[f_{1}, \ldots, f_{r}, L_{s}^{(\alpha)}\right],
$$

where we assume that $s \notin\left\{n_{1}, \ldots, n_{r_{1}}\right\}$. Up to a possible sign factor, this polynomial is the generalized Laguerre polynomial of parameter $\alpha$ associated with the partitions $\tilde{\lambda}$ and $\mu$, where $\tilde{\lambda}$ is the partition corresponding to the degrees $n_{1}, \ldots, n_{r_{1}}$, and $s$. Because $|\tilde{\lambda}|=|\lambda|+s-r_{1}$, we have, by Lemma 1, that the degree of (15) is $s+|\lambda|+|\mu|-r_{1}$. By varying $s$, we obtain polynomials of degrees that are in the degree sequence associated with $\lambda$ and $\mu$ that is defined as follows.

Definition 3. The degree sequence associated with partitions $\lambda$ and $\mu$ is

$$
\begin{aligned}
\mathbb{N}_{\lambda, \mu}= & \left\{n \in \mathbb{N} \cup\{0\}|n \geq| \lambda|+| \mu \mid-r_{1} \text { and } n-|\lambda|-|\mu| \neq \lambda_{j}-j\right. \\
& \text { for } \left.j=1, \ldots, r_{1}\right\} .
\end{aligned}
$$

For $n \in \mathbb{N}_{\lambda, \mu}$, we take $s=n-|\lambda|-|\mu|+r_{1}$ and this is a nonnegative integer because of the first condition in (16). The second condition is such that $s \neq n_{j}$ for every $j=1, \ldots, r_{1}$. This then leads to the following definition of the exceptional Lauerre polynomials.

DeFINITION 4. The exceptional Laguerre polynomials of parameter $\alpha$ associated with the two partitions $\lambda$ and $\mu$ are given by

$$
L_{\lambda, \mu, n}^{(\alpha)}(x)=e^{-r_{2} x} \cdot \operatorname{Wr}\left[f_{1}, \ldots, f_{r}, L_{s}^{(\alpha)}\right], \quad n \in \mathbb{N}_{\lambda, \mu},
$$

where $s=n-|\lambda|-|\mu|+r_{1}$ and $f_{1}, \ldots, f_{r}$ are as in (8) and (9).

The definition is such that $L_{\lambda, \mu, n}^{(\alpha)}$ has degree $n$. There are $|\lambda|+|\mu|$ degrees that do not occur, namely, those nonnegative integers outside of $\mathbb{N}_{\lambda, \mu}$. They are called the exceptional degrees. The leading coefficient of $L_{\lambda, \mu, n}^{(\alpha)}$ can be determined as in Lemma 1.

Remark 2. When both partitions are empty we obtain that $L_{\emptyset, \emptyset, n}^{(\alpha)}(x)=$ $L_{n}^{(\alpha)}(x)$ for all $x \in \mathbb{C}$. Hence, the exceptional Laguerre polynomial is a generalization of the usual Laguerre polynomial.

Remark 3. Similarly to (17), we can define another exceptional Laguerre polynomial by

$$
\tilde{L}_{\lambda, \mu, n}^{(\alpha)}(x)=e^{-\left(r_{2}+1\right) x} \cdot \operatorname{Wr}\left[f_{1}, \ldots, f_{r}, e^{x} L_{s}^{(\alpha)}(-x)\right],
$$

where we take $s=n-|\lambda|-|\mu|+r_{2} \geq 0$, and $s \neq m_{j}$ for $j=1, \ldots, r_{2}$. Using the duality from Lemma 3 , we can reduce this to the case (17) 
because

$$
\tilde{L}_{\lambda, \mu, n}^{(\alpha)}(x)=(-1)^{\frac{r_{1}\left(r_{1}+1\right)}{2}+\frac{r_{2}\left(r_{2}+1\right)}{2}} L_{\mu, \lambda, n}^{(\alpha)}(-x) .
$$

If $\alpha>-1$ and the partition $\lambda$ is even, then the exceptional Laguerre polynomials form a complete set of orthogonal polynomials on the positive real line. This result is due to Durán and Pérez $[2,17]$.

Lemma 6. Suppose $\alpha>-1$. If $\lambda$ is an even partition, then the polynomials $L_{\lambda, \mu, n}^{(\alpha)}$ for $n \in \mathbb{N}_{\lambda, \mu}$ are orthogonal on $[0, \infty)$ with respect to the positive weight function

$$
W_{\lambda, \mu}^{(\alpha)}(x)=\frac{x^{\alpha+r} e^{-x}}{\left(\Omega_{\lambda, \mu}^{(\alpha)}(x)\right)^{2}}, \quad x>0 .
$$

That is, if $n, m \in \mathbb{N}_{\lambda, \mu}$ with $n \neq m$, then

$$
\int_{0}^{\infty} L_{\lambda, \mu, n}^{(\alpha)}(x) L_{\lambda, \mu, m}^{(\alpha)}(x) W_{\lambda, \mu}^{(\alpha)}(x) d x=0 .
$$

Moreover, they form a complete orthogonal set in $L^{2}\left([0, \infty), W_{\lambda, \mu}^{(\alpha)}(x) d x\right)$.

The conditions $\alpha>-1$ and $\lambda$ an even partition are not best possible. More general, but less easy to state, conditions can also be found in $[2,17]$.

Remark 4. In the literature, one often refers to exceptional Laguerre polynomials when these polynomials form an orthogonal complete set. In our set-up, we defined a set of polynomials in (17) and named them as exceptional Laguerre polynomials for every two partitions $\lambda$ and $\mu$ and for every choice of parameter $\alpha$. For us, the exceptional Laguerre polynomials form an orthogonal complete set of polynomials only in certain special cases, such as given by Lemma 6 .

\section{The degree and leading coefficient of $\boldsymbol{\Omega}_{\lambda, \mu}^{(\alpha)}$}

In this section, we give an alternative proof of Lemma 1. It follows from the subsequent more general result that holds for arbitrary polynomials, and not just for Laguerre polynomials.

PROPOSITION 1. Let $r_{1}, r_{2}$ be nonnegative integers and define $r=r_{1}+r_{2}$. Let $R_{1}, \ldots, R_{r}$ be nonzero polynomials such that $\operatorname{deg} R_{i} \neq \operatorname{deg} R_{j}$ whenever 
$i \neq j$ and $1 \leq i, j \leq r_{1}$ or $r_{1}+1 \leq i, j \leq r$. Then the polynomial

$$
\Omega(x)=e^{-r_{2} x} \cdot \operatorname{Wr}\left[R_{1}, \ldots, R_{r_{1}}, e^{x} R_{r_{1}+1}, \ldots, e^{x} R_{r}\right]
$$

has degree

$$
\operatorname{deg} \Omega=\sum_{i=1}^{r} \operatorname{deg} R_{i}-\left(\begin{array}{c}
r_{1} \\
2
\end{array}\right)-\left(\begin{array}{c}
r_{2} \\
2
\end{array}\right) .
$$

Moreover, if all polynomials $R_{i}$ are monic, then the leading coefficient of $\Omega$ is given by

$$
\prod_{1 \leq i<j \leq r_{1}}\left(\operatorname{deg} R_{j}-\operatorname{deg} R_{i}\right) \prod_{1 \leq i<j \leq r_{2}}\left(\operatorname{deg} R_{r_{1}+j}-\operatorname{deg} R_{r_{1}+i}\right) .
$$

We use the notation

$$
\begin{aligned}
\Omega & =\Omega\left(R_{1}, \ldots R_{r_{1}} ; R_{r_{1}+1}, \ldots, R_{r}\right) \\
& =e^{-r_{2} x} \cdot \operatorname{Wr}\left[R_{1}, \ldots, R_{r_{1}}, e^{x} R_{r_{1}+1}, \ldots, e^{x} R_{r}\right] .
\end{aligned}
$$

The proof is by induction on the total sum of the degrees, and in the induction we use the following lemma.

Lemma 7. Let $R_{1}, \ldots, R_{r}$ be polynomials, not necessarily of distinct degrees. Then,

$$
\begin{aligned}
& \frac{d}{d x} \Omega\left(R_{1}, \ldots R_{r_{1}} ; R_{r_{1}+1}, \ldots, R_{r}\right)=\Omega\left(R_{1}^{\prime}, \ldots, R_{r_{1}} ; R_{r_{1}+1}, \ldots, R_{r}\right) \\
& \quad+\cdots+\Omega\left(R_{1}, \ldots, R_{r_{1}}^{\prime} ; R_{r_{1}+1}, \ldots, R_{r}\right)+\Omega\left(R_{1}, \ldots, R_{r_{1}} ; R_{r_{1}+1}^{\prime}, \ldots, R_{r}\right) \\
& \quad+\cdots+\Omega\left(R_{1}, \ldots, R_{r_{1}} ; R_{r_{1}+1}, \ldots, R_{r}^{\prime}\right) .
\end{aligned}
$$

Proof. Because the Wronskian is multilinear in its arguments, we can compute from (23)

$$
\begin{aligned}
\frac{d}{d x} \Omega= & -r_{2} \Omega+e^{-r_{2} x} \cdot \frac{d}{d x} \operatorname{Wr}\left[R_{1}, \ldots, R_{r_{1}}, e^{x} R_{r_{1}+1}, \ldots, e^{x} R_{r}\right] \\
= & -r_{2} \Omega+\Omega\left(R_{1}^{\prime}, \ldots, R_{r_{1}} ; R_{r_{1}+1}, \ldots, R_{r}\right)+\cdots \\
& +\Omega\left(R_{1}, \ldots, R_{r_{1}}^{\prime} ; R_{r_{1}+1}, \ldots, R_{r}\right) \\
& +\Omega\left(R_{1}, \ldots, R_{r_{1}} ; R_{r_{1}+1}+R_{r_{1}+1}^{\prime}, \ldots, R_{r}\right)+\cdots \\
& +\Omega\left(R_{1}, \ldots, R_{r_{1}} ; R_{r_{1}+1}, \ldots, R_{r}+R_{r}^{\prime}\right) .
\end{aligned}
$$

From the multilinearity of $\Omega$ with respect to each of its arguments, we then obtain (24).

As a second preparation for the proof of Proposition (1), we state and prove an identity that will be used to establish the formula (22) for the 
leading coefficient of $\Omega$. We need the identity (25) for natural numbers, but as the proof shows it is valid for arbitrary real or complex numbers.

LEMMA 8. Let $x_{1}, \ldots, x_{r}$ be $r$ pairwise different numbers, i.e., $x_{i} \neq x_{j}$ for $i \neq j$. Then the following holds,

$$
\sum_{k=1}^{r}\left(x_{k} \prod_{\substack{j=1 \\
j \neq k}}^{r} \frac{x_{j}-\left(x_{k}-1\right)}{x_{j}-x_{k}}\right)=\sum_{k=1}^{r} x_{k}-\left(\begin{array}{l}
r \\
2
\end{array}\right) .
$$

Proof. We prove this by induction on $r$. When $r=1$, the identity is trivially true. Thus, take $r>1$. We claim that

$$
\sum_{k=1}^{r} x_{k} \prod_{\substack{j=1 \\ j \neq k}}^{r} \frac{x_{j}-x_{k}+1}{x_{j}-x_{k}}=\sum_{k=1}^{r-1} x_{k} \prod_{\substack{j=1 \\ j \neq k}}^{r-1} \frac{x_{j}-x_{k}+1}{x_{j}-x_{k}}+x_{r}-(r-1)
$$

from which the identity (25) follows by applying the induction hypothesis on the right-hand side of the equality. Thus, it remains to show that (26) holds true. We start by splitting the sum into two parts

$$
\sum_{k=1}^{r} x_{k} \prod_{\substack{j=1 \\ j \neq k}}^{r} \frac{x_{j}-x_{k}+1}{x_{j}-x_{k}}=x_{r} \prod_{j=1}^{r-1} \frac{x_{j}-x_{r}+1}{x_{j}-x_{r}}+\sum_{k=1}^{r-1} x_{k} \prod_{\substack{j=1 \\ j \neq k}}^{r} \frac{x_{j}-x_{k}+1}{x_{j}-x_{k}}
$$

Consider $x_{r}$ as a variable, we then have the partial fraction decomposition

$$
x_{r} \prod_{j=1}^{r-1} \frac{x_{j}-\left(x_{r}-1\right)}{x_{j}-x_{r}}=B x_{r}+C+\sum_{k=1}^{r-1} \frac{A_{k}}{x_{r}-x_{k}}
$$

for some nonzero constants $B, C$, and

$$
A_{k}=-x_{k} \frac{\prod_{j=1}^{r-1}\left(x_{j}-x_{k}+1\right)}{\prod_{\substack{j=1 \\ j \neq k}}^{r-1}\left(x_{j}-x_{k}\right)}, \quad k=1, \ldots, r-1 .
$$

From the fact that

$$
\frac{x_{j}-\left(x_{r}-1\right)}{x_{j}-x_{r}}=1+\frac{1}{x_{j}-x_{r}}
$$

we see that

$$
\prod_{j=1}^{r-1} \frac{x_{j}-\left(x_{r}-1\right)}{x_{j}-x_{r}}=1+\sum_{j=1}^{r-1} \frac{1}{x_{j}-x_{r}}+O\left(\frac{1}{x_{r}^{2}}\right)
$$


and thus $B=1$ and $C=-(r-1)$. Therefore, we can rewrite (28) as

$$
x_{r} \prod_{j=1}^{r-1} \frac{x_{j}-\left(x_{r}-1\right)}{x_{j}-x_{r}}=-\sum_{k=1}^{r-1} x_{k} \frac{\prod_{\substack{j=1 \\ j=1 \\ j \neq k}}^{r-1}\left(x_{j}-x_{k}+1\right)}{\prod_{j}^{r}\left(x_{j}-x_{k}\right)}+x_{r}-(r-1) .
$$

If we now look at the product $\prod_{j=1}^{r-1}\left(x_{j}-x_{k}+1\right)$, it is clear that this is the same as $j$ runs from 1 to $r-1$ excluding $k$ as for $j=k$ we have that $x_{j}+x_{k}+1=1$. Moreover, as

$$
\frac{1}{x_{r}-x_{k}+1}=1-\frac{x_{r}-x_{k}}{x_{r}-x_{k}+1},
$$

by adding $j=r$ in the product, we can rewrite (29) as

$$
\begin{aligned}
x_{r} \prod_{j=1}^{r-1} \frac{x_{j}-\left(x_{r}-1\right)}{x_{j}-x_{r}}= & -\sum_{k=1}^{r-1} x_{k} \prod_{\substack{j=1 \\
j \neq k}}^{r} \frac{x_{j}-x_{k}+1}{x_{j}-x_{k}} \\
& +\sum_{k=1}^{r-1} x_{k} \prod_{\substack{j=1 \\
j \neq k}}^{r-1} \frac{x_{j}-x_{k}+1}{x_{j}-x_{k}}+x_{r}-(r-1) .
\end{aligned}
$$

If we plug this value in (27), we obtain (26) which ends the proof of the lemma.

Now, we are ready for the proof of Proposition 1.

Proof of Proposition 1. Let $R_{1}, \ldots, R_{r}$ be an arbitrary sequence of monic polynomials, and let $\Omega=\Omega\left(R_{1}, \ldots, R_{r_{1}}, R_{r_{1}+1}, \ldots, R_{r}\right)$ be as in (20). We are going to prove that

$$
\operatorname{deg} \Omega \leq \sum_{i=1}^{r} \operatorname{deg} R_{i}-\left(\begin{array}{c}
r_{1} \\
2
\end{array}\right)-\left(\begin{array}{c}
r_{2} \\
2
\end{array}\right)
$$

with equality if and only if the degree condition of Proposition 1 is satisfied. In that case, we show that the leading coefficient is given by (22). If $\Omega \equiv 0$, then we take $\operatorname{deg} \Omega=-\infty$.

If $R_{1}, \ldots, R_{r_{1}}$ or $R_{r_{1}+1}, \ldots, R_{r}$ are linearly dependent, then $\Omega \equiv 0$ and then (30) is automatically satisfied. Thus, we assume that $R_{1}, \ldots, R_{r_{1}}$ are linearly independent, as well as $R_{r_{1}+1}, \ldots, R_{r}$. By permuting entries in the Wronskian, we may also assume that

$$
\begin{array}{ll}
\operatorname{deg} R_{i} \leq \operatorname{deg} R_{i+1}, & \text { for } i=1, \ldots, r_{1}-1, \\
\operatorname{deg} R_{r_{1}+i} \leq \operatorname{deg} R_{r_{1}+i+1}, & \text { for } i=1, \ldots, r_{2}-1 .
\end{array}
$$


Another choice would be that the degrees are decreasing as we did so far. However, the choice we make does not influence the result of the proposition as the leading coefficient (22) captures this choice.

We will then use induction on the number $N=\sum_{k=1}^{r} \operatorname{deg} R_{k}-\left(\begin{array}{c}r_{1} \\ 2\end{array}\right)-\left(\begin{array}{c}r_{2} \\ 2\end{array}\right)$. Under the above assumptions, the smallest possible number $N=0$ is reached when $\operatorname{deg} R_{i}=i-1$ for $i=1, \ldots, r_{1}-1$ and $\operatorname{deg} R_{r_{1}+i}=i-1$ for $i=1, \ldots, r_{2}-1$. The first $r_{1}$ columns in the Wronskian (20) then have an upper triangular form and we find by expanding

$$
\Omega=\left(\prod_{i=1}^{r_{1}}\left(\operatorname{deg} R_{i}\right) !\right) e^{-r_{2} x} \cdot \operatorname{Wr}\left[e^{x} S_{1}, \ldots, e^{x} S_{r_{2}}\right]
$$

where $S_{j}=e^{-x}\left(\frac{d}{d x}\right)^{r_{1}}\left(e^{x} R_{r_{1}+j}\right)$. Note that $S_{j}$ is a monic polynomial with $\operatorname{deg} S_{j}=\operatorname{deg} R_{r_{1}+j}=j-1$ for $j=1, \ldots, r_{2}$. Using (12), we have

$$
e^{-r_{2} x} \cdot \operatorname{Wr}\left[e^{x} S_{1}, \ldots, e^{x} S_{r_{2}}\right]=\operatorname{Wr}\left[S_{1}, \ldots, S_{r_{2}}\right]=\prod_{j=1}^{r_{2}}\left(\operatorname{deg} R_{r_{1}+j}\right) ! .
$$

Thus,

$$
\Omega=\prod_{i=1}^{r_{1}}\left(\operatorname{deg} R_{i}\right) ! \prod_{j=1}^{r_{2}}\left(\operatorname{deg} R_{r_{1}+j}\right) !
$$

which is a constant, so that the degree condition (21) is satisfied. Also, the constant is equal to (22), as can be easily verified. This completes the proof of the base step of the induction.

In the induction step, we take $N>0$ and we assume that the statement is true whenever the sum of the degrees of the polynomials is at most $N-1+\left(\begin{array}{c}r_{1} \\ 2\end{array}\right)+\left(\begin{array}{c}r_{2} \\ 2\end{array}\right)$.

We take polynomials $R_{1}, \ldots, R_{r}$ with $\sum_{k=1}^{r} R_{k}=N+\left(\begin{array}{c}r_{1} \\ 2\end{array}\right)+\left(\begin{array}{c}r_{2} \\ 2\end{array}\right)$. We assume $R_{1}, \ldots, R_{r_{1}}$ and $R_{r_{1}+1}, \ldots, R_{r_{2}}$ are linearly independent and without loss of generality we also assume (31). If equality holds somewhere in (31), then we perform a column operation on the Wronskian to reduce the degree of one of the polynomials. Then, from the induction hypothesis, it follows that (30) holds with strict inequality. The coefficient of $x^{N}$ is thus zero, and this agrees with the formula (22).

Thus, we may assume

$$
\begin{array}{ll}
\operatorname{deg} R_{i}<\operatorname{deg} R_{i+1}, & \text { for } i=1, \ldots, r_{1}-1, \\
\operatorname{deg} R_{r_{1}+i}<\operatorname{deg} R_{r_{1}+i+1}, & \text { for } i=1, \ldots, r_{2}-1 .
\end{array}
$$

The identity (24) expresses $\frac{d}{d x} \Omega\left(R_{1}, \ldots, R_{r_{1}} ; R_{r_{1}+1}, \ldots, R_{r_{2}}\right)$ as as sum of $r$ terms, each of which is an $\Omega$-polynomial built out of polynomials whose total degree is $N-1+\left(\begin{array}{c}r_{1} \\ 2\end{array}\right)+\left(\begin{array}{c}r_{2} \\ 2\end{array}\right)$. According to the induction hypothesis, 
the degree of each of these terms is at most $N-1$. Thus, $\operatorname{deg} \Omega^{\prime} \leq N-1$ and (30) follows after an integration step.

To determine the coefficient of $x^{N}$, we first compute the coefficient of $x^{N-1}$ in each of the terms in the right-hand side of (24). We denote $x_{k}=\operatorname{deg} R_{k}$ for $k=1, \ldots, r_{1}$, and $y_{k}=\operatorname{deg} R_{r_{1}+k}$ for $k=1, \ldots, r_{2}$. By the induction hypothesis, the coefficient of $x^{N-1}$ of the $k$ th term is

$$
x_{k} \prod_{\substack{j=1 \\ j \neq k}}^{r_{1}} \frac{x_{j}-\left(x_{k}-1\right)}{x_{j}-x_{k}} \prod_{1 \leq i<j \leq r_{1}}\left(x_{j}-x_{i}\right) \prod_{1 \leq i<j \leq r_{2}}\left(y_{j}-y_{i}\right), \quad k=1, \ldots, r_{1},
$$

while the coefficient for the $\left(r_{1}+k\right)$ th term is

$$
y_{k} \prod_{\substack{j=1 \\ j \neq k}}^{r_{1}} \frac{y_{j}-\left(y_{k}-1\right)}{y_{j}-y_{k}} \prod_{1 \leq i<j \leq r_{1}}\left(x_{j}-x_{i}\right) \prod_{1 \leq i<j \leq r_{2}}\left(y_{j}-y_{i}\right), \quad k=1, \ldots, r_{2} .
$$

Note that (33) is also valid if $\operatorname{deg} R_{1}=0$, or if $\operatorname{deg} R_{k}^{\prime}=\operatorname{deg} R_{k-1}$, for some $k=2, \ldots, r_{1}$, because in these cases (33) vanishes, as it should. Likewise, (34) is also valid if $\operatorname{deg} R_{r_{1}+1}=0$, or if $\operatorname{deg} R_{r_{1}+k}^{\prime}=\operatorname{deg} R_{r_{1}+k}$ for some $k=2, \ldots, r_{2}$.

Adding (33) and (34), and using Lemma 8, we find that the coefficient of $x^{N-1}$ in $\Omega^{\prime}$ is

$$
\left(\sum_{k=1}^{r_{1}} x_{k}-\left(\begin{array}{c}
r_{1} \\
2
\end{array}\right)+\sum_{k=1}^{r_{2}} y_{k}-\left(\begin{array}{c}
r_{2} \\
2
\end{array}\right)\right) \prod_{1 \leq i<j \leq r_{1}}\left(x_{j}-x_{i}\right) \prod_{1 \leq i<j \leq r_{2}}\left(y_{j}-y_{i}\right) .
$$

Now, recall

$$
\sum_{k=1}^{r_{1}} x_{k}+\sum_{k=1}^{r_{2}} y_{k}=\sum_{k=1}^{r} \operatorname{deg} R_{k}=N+\left(\begin{array}{c}
r_{1} \\
2
\end{array}\right)+\left(\begin{array}{c}
r_{2} \\
2
\end{array}\right) .
$$

Therefore, the coefficient of $x^{N-1}$ of $\Omega^{\prime}$ is

$$
N \prod_{1 \leq i<j \leq r_{1}}\left(x_{j}-x_{i}\right) \prod_{1 \leq i<j \leq r_{2}}\left(y_{j}-y_{i}\right),
$$

and after integration we find that the coefficient of $x^{N}$ of $\Omega$ is equal to (22). The coefficient is nonzero and therefore $\Omega$ has degree $N$ as claimed in (21). This completes the proof of the induction step, and Proposition 1 is proved.

Using similar ideas, we can prove the following proposition that we will use in Lemma 10. It applies to a more general situation, but it only gives an upper bound on the degree of the polynomial, and it does not give information on the leading coefficient. 
PROPOSITION 2. Take two nonnegative integers $r_{1}, r_{2}$ and define $r=$ $r_{1}+r_{2}$. Let $R_{1}, \ldots, R_{r}$ be polynomials. Take nonnegative integers $0 \leq l_{1}<$ $l_{2}<\cdots<l_{r}$. Consider the polynomial

$$
Q(x)=e^{-r_{2} x}\left|\begin{array}{ccccc}
R_{1}^{\left(l_{1}\right)} & \ldots & R_{r_{1}}^{\left(l_{1}\right)} & \left(e^{x} R_{r_{1}+1}\right)^{\left(l_{1}\right)} \ldots & \left(e^{x} R_{r}\right)^{\left(l_{1}\right)} \\
R_{1}^{\left(l_{2}\right)} & \ldots & R_{r_{1}}^{\left(l_{2}\right)} & \left(e^{x} R_{r_{1}+1}\right)^{\left(l_{2}\right)} \cdots & \left(e^{x} R_{r}\right)^{\left(l_{2}\right)} \\
\vdots & \vdots & \ddots & \vdots & \\
R_{1}^{\left(l_{r}\right)} & \ldots & R_{r_{1}}^{\left(l_{r}\right)} & \left(e^{x} R_{r_{1}+1}\right)^{\left(l_{r}\right)} \ldots & \left(e^{x} R_{r}\right)^{\left(l_{r}\right)}
\end{array}\right| .
$$

Then, the degree of $Q$ is at most $\sum_{i=1}^{r} \operatorname{deg} R_{i}-\sum_{i=1}^{r_{1}} l_{i}-\left(\begin{array}{c}r_{2} \\ 2\end{array}\right)$.

Proof. The proof is similar to (part of) the proof of Proposition 1. We only need to observe that similar to (24), we now have

$$
\begin{aligned}
Q^{\prime}= & Q\left(R_{1}^{\prime}, \ldots, R_{r_{1}} ; R_{r_{1}+1}, \ldots, R_{r}\right)+\cdots+Q\left(R_{1}, \ldots, R_{r_{1}}^{\prime} ; R_{r_{1}+1}, \ldots, R_{r}\right) \\
& +Q\left(R_{1}, \ldots, R_{r_{1}} ; R_{r_{1}+1}^{\prime}, \ldots, R_{r}\right)+\cdots+Q\left(R_{1}, \ldots, R_{r_{1}} ; R_{r_{1}+1}, \ldots, R_{r}^{\prime}\right)
\end{aligned}
$$

with the (hopefully obvious) notation that $Q\left(R_{1}, \ldots, R_{r_{1}} ; R_{r_{1}+1}, \ldots, R_{r}\right)$ is the polynomial (35) based on the polynomials $R_{1}, \ldots, R_{r}$, and with the same sequence $l_{1}<l_{2}<\cdots<l_{r}$.

\section{Construction of the generalized Laguerre polynomial}

In this section, we will discuss the construction of the generalized Laguerre polynomial. In particular, we show that it is sufficient to include only the first two types of eigenfunctions of Table 1 in the Wronskian. To this end, we start from a Wronskian including all four types and show that, up to a constant, this equals a Wronskian containing only the first two types as in (7). Hence, in the general setup, we start from eigenfunctions $f_{1}, \ldots, f_{r}$ of the Laguerre differential operator (2) where

$$
\begin{gathered}
f_{j}(x)=L_{n_{j}}^{(\alpha)}(x), \quad j=1, \ldots, r_{1}, \\
f_{r_{1}+j}(x)=e^{x} L_{m_{j}}^{(\alpha)}(-x), \quad j=1, \ldots, r_{2}, \\
f_{r_{1}+r_{2}+j}(x)=x^{-\alpha} L_{m_{j}^{\prime}}^{(-\alpha)}(x), \quad j=1, \ldots, r_{3}, \\
f_{r_{1}+r_{2}+r_{3}+j}(x)=e^{x} x^{-\alpha} L_{n_{j}^{\prime}}^{(-\alpha)}(-x), \quad j=1, \ldots, r_{4},
\end{gathered}
$$

with $\quad r_{1}+r_{2}+r_{3}+r_{4}=r, \quad n_{1}>n_{2}>\cdots>n_{r_{1}} \geq 0, \quad m_{1}>m_{2}>\cdots>$ $m_{r_{2}} \geq 0, m_{1}^{\prime}>m_{2}^{\prime}>\cdots>m_{r_{3}}^{\prime} \geq 0$, and $n_{1}^{\prime}>n_{2}^{\prime}>\cdots>n_{r_{1}}^{\prime} \geq 0$. The result of this section is that there are partitions $\lambda$ and $\mu$, an integer $t$, and a constant $C$ such that

$$
e^{-\left(r_{2}+r_{4}\right) x} x^{\left(\alpha+r_{1}+r_{2}\right)\left(r_{3}+r_{4}\right)} \cdot \operatorname{Wr}\left[f_{1}, f_{2}, \ldots, f_{r}\right](x)=C \Omega_{\lambda, \mu}^{(\alpha-t)}(x) .
$$


The fact that we only need two types of eigenfunctions instead of all four possibilities is essentially stated in for example [12,14,34]. However, explicit equalities were never derived. In [34], Takemura discusses the reduction in the Jacobi case and concludes that similar methods must work for the Laguerre case as well. We derive these explicit identities in this section. We use the same ideas to describe this procedure as in the recent paper [31] where the authors discuss pseudo-Wronskians of Hermite polynomials.

To describe how $\lambda, \mu$, and $t$ are obtained from all the indices, it is useful to introduce Maya diagrams.

\subsection{Maya diagrams}

A Maya diagram $M$ is a subset of the integers that contains a finite number of positive integers and excludes a finite number of negative integers. We visualize it as an infinite row of boxes which are empty or filled. We order these boxes by corresponding them to the set of integers and therefore we define an origin. To the right of the origin, there are only finitely many filled boxes. Each of these filled boxes corresponds to a nonnegative integer $n \geq 0$. All filled boxes to the right of the origin are labeled by a finite decreasing sequence

$$
n_{1}>n_{2}>\cdots>n_{r_{1}} \geq 0,
$$

where $r_{1}$ is the number of filled boxes to the right of the origin. If $r_{1}=0$, then the sequence is empty.

To the left of the origin, there are only finitely many empty boxes. Each empty box corresponds to a negative integer $k<0$. We link this negative integer to a nonnegative integer $n^{\prime}=-k-1 \geq 0$. We obtain a second finite decreasing sequence

$$
n_{1}^{\prime}>n_{2}^{\prime}>\cdots>n_{r_{4}}^{\prime} \geq 0
$$

which labels the positions of the empty boxes to the left of the origin, and $r_{4}$ is the number of those boxes. The Maya diagram is encoded by these two sequences

$$
M: \quad\left(n_{1}^{\prime}, n_{2}^{\prime}, \ldots, n_{r_{4}}^{\prime} \mid n_{1}, n_{2}, \ldots, n_{r_{1}}\right) .
$$

For example, consider the following Maya diagram $M$.

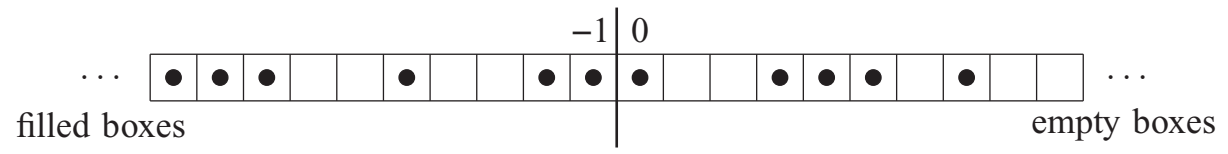

The boxes on the right correspond to the finite decreasing sequence $(7,5,4,3,0)$. The empty boxes on the left likewise correspond to $(6,5,3,2)$, and therefore 


$$
M: \quad(6,5,3,2 \mid 7,5,4,3,0) .
$$

Remark 5. In Theorem 1, we extend the decreasing sequence $n_{1}>n_{2}>$ $\cdots>n_{r_{1}}$ to an infinite decreasing sequence $n_{1}>n_{2}>\cdots>n_{r_{1}}>n_{r_{1}+1}>$ $n_{r_{1}+2}>\cdots$ giving the positions of all the filled boxes. Thus, if $j \geq r_{1}+1$, then $n_{j}<0$. In our example, we get $(7,5,4,3,0$, $-1,-2,-5,-8,-9,-10, \cdots)$.

We say that a Maya diagram $\tilde{M}$ is equivalent to $M$ if $\tilde{M}$ is obtained from $M$ by moving the position of the origin. Hence, the sequence of filled and empty boxes remains unchanged. For example, the Maya diagram $\tilde{M}$

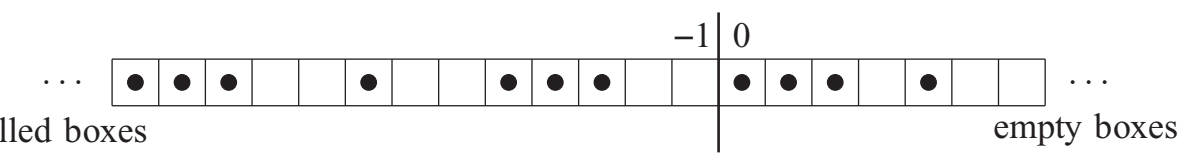

is equivalent to $M$ because $\tilde{M}=M-3$, i.e., the origin is moved three steps to the right. In this example, $\tilde{M}$ is given by

$$
\tilde{M}: \quad(9,8,6,5,1,0 \mid 4,2,1,0) \text {. }
$$

It is clear from this example that the total length of both sequences in the encoding of equivalent Maya diagrams needs not be the same. The total length of $M$ is 9 , while it is 10 for $\tilde{M}$.

A canonical choice for the position of the origin is to have it such that all boxes to the left are filled, while the first box to the right is empty. In the example, it would be

filled boxes

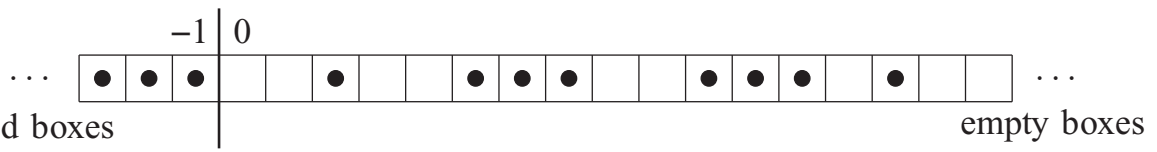

with the encoding

$$
\tilde{M}=M+7: \quad(\emptyset \mid 14,12,11,10,7,6,5,2) .
$$

Because there are no empty boxes on the left, we only have one decreasing sequence, say $\left(\emptyset \mid n_{1}, n_{2}, \ldots, n_{r}\right)$ with $n_{r} \geq 1$. It is associated with a partition as before, which we denote it by

$$
\lambda=\lambda(M) \text {. }
$$

In our example, $\lambda(M)=(7,6,6,6,4,4,4,2)$.

We use

$$
t=t(M)
$$

to denote the shift $M \rightarrow \tilde{M}=M+t$ that we have to apply to $M$ to take it into this canonical form. If $M$ is encoded by (40) with $r_{4} \geq 1$, then

$$
t(M)=n_{1}^{\prime}+1
$$


and in that case $t(M) \geq 1$. If $r_{4}=0$, then $t(M) \leq 0$.

Another possible choice for the position of the origin is to have it in such a way that the number of empty boxes to the left is the same as the number of filled boxes to the right. It is easy to see that there is a unique such position and in our example it is

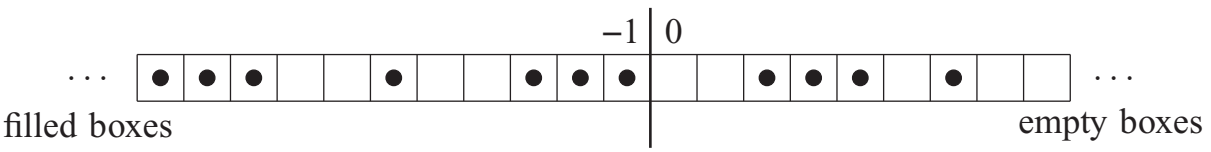

with encoding

$$
M-1:(7,6,4,3 \mid 6,4,3,2) .
$$

This coincides with the Frobenius representation of the partition $\lambda(M)$.

We can also put the origin so that all the boxes to the right are empty, and the first box to the left is filled. In the example, it is

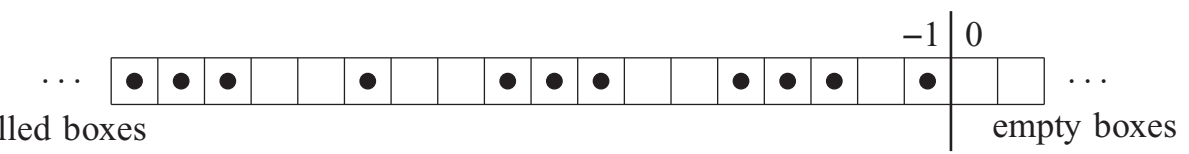

This has an encoding $\left(n_{1}^{\prime}, n_{2}^{\prime}, \ldots, n_{s}^{\prime} \mid \emptyset\right)$ with $n_{s}^{\prime} \geq 1$. Then,

$$
\lambda_{j}^{\prime}=n_{j}^{\prime}-s+j, \quad j=1, \ldots, s
$$

is the partition that is conjugate to $\lambda$, see (14).

This is all we need about Maya diagrams. A more thorough introduction to Maya diagrams can be found in for example [31]. More information about the Frobenius representation is covered in [35].

\subsection{The result}

Now, we are able to state the main result of this section about the construction of the generalized Laguerre polynomial.

THEOREM 1. Let $f_{1}, \ldots, f_{r}$ be as in (36)-(39), and let

$$
\begin{array}{ll}
M_{1}: & \left(n_{1}^{\prime}, \ldots, n_{r_{4}}^{\prime} \mid n_{1}, \ldots, n_{r_{1}}\right), \\
M_{2}: & \left(m_{1}^{\prime}, \ldots, m_{r_{3}}^{\prime} \mid m_{1}, \ldots, m_{r_{2}}\right),
\end{array}
$$

be two Maya diagrams built out of the degrees of the Laguerre polynomials appearing in (36)-(39). Let $\lambda=\lambda\left(M_{1}\right)$ and $\mu=\lambda\left(M_{2}\right)$ be the two partitions that are associated with $M_{1}$ and $M_{2}$ as described above, and let $t_{1}=t\left(M_{1}\right)$, $t_{2}=t\left(M_{2}\right)$ as in (42).

Then, there is a constant $C=C\left(\alpha, M_{1}, M_{2}\right)$ such that

$$
e^{-\left(r_{2}+r_{4}\right) x} x^{\left(\alpha+r_{1}+r_{2}\right)\left(r_{3}+r_{4}\right)} \cdot \operatorname{Wr}\left[f_{1}, \ldots, f_{r}\right](x)=C \Omega_{\lambda, \mu}^{\left(\alpha-t_{1}-t_{2}\right)}(x)
$$


where the constant $C$ is given by

$$
\begin{aligned}
C\left(\alpha, M_{1}, M_{2}\right)= & (-1)^{d} \prod_{j=1}^{r_{1}} \prod_{k=1}^{r_{3}}\left(m_{k}^{\prime}-\alpha-n_{j}\right) \prod_{j=1}^{r_{2}} \prod_{k=1}^{r_{4}}\left(n_{k}^{\prime}-\alpha-m_{j}\right) \\
& \times \prod_{j=1}^{r_{1}} \prod_{k=1}^{r_{4}}\left(n_{j}+n_{k}^{\prime}+1\right) \prod_{j=1}^{r_{2}} \prod_{k=1}^{r_{3}}\left(m_{j}+m_{k}^{\prime}+1\right),
\end{aligned}
$$

and

$$
d= \begin{cases}d_{0} & \text { if } r_{3}=r_{4}=0 \\ d_{0}+d_{1} & \text { if } r_{3}=0 \text { and } r_{4} \geq 1 \\ d_{2} & \text { if } r_{3} \geq 1 \text { and } r_{4}=0 \\ d_{1}+d_{2} & \text { if } r_{3} \geq 1 \text { and } r_{4} \geq 1\end{cases}
$$

with

$$
\begin{aligned}
& d_{0}=\left|t_{2}\right| r_{2}-\frac{\left|t_{2}\right|\left(\left|t_{2}\right|+1\right)}{2} \\
& d_{1}=\frac{r_{4}\left(r_{4}-1\right)}{2}+r_{1} r_{4}+\frac{\left(n_{1}^{\prime}+1-r_{4}\right)\left(n_{1}^{\prime}+2+r_{4}\right)}{2}+\sum_{i=1}^{n_{1}^{\prime}+1-r_{4}} n_{r_{1}+i}, \\
& d_{2}=r_{2}\left(m_{1}^{\prime}+1\right)+\left(m_{1}^{\prime}+1\right)\left(m_{1}^{\prime}+1-r_{3}\right)+\sum_{i=1}^{m_{1}^{\prime}+1-r_{3}} m_{r_{2}+i} .
\end{aligned}
$$

Here, we used the infinite decreasing sequences $\left(n_{i}\right)_{i=1}^{\infty}$ and $\left(m_{i}\right)_{i=1}^{\infty}$ which give the positions of all filled boxes, see Remark 5.

The constant (44) is nonzero, if and only if

$$
\begin{array}{ll}
\alpha \neq m_{i}^{\prime}-n_{j}, & \text { for } i=1, \ldots, r_{3}, \quad j=1, \ldots, r_{1}, \\
\alpha \neq n_{i}^{\prime}-m_{j}, & \text { for } i=1, \ldots, r_{4}, \quad j=1, \ldots, r_{2} .
\end{array}
$$

Indeed, if $\alpha$ does not satisfy these conditions, $\Omega_{M_{1}, M_{2}}^{(\alpha)}$ vanishes identically because in such a case, two functions in the Wronskian are multiples of each other and therefore the Wronskian is zero. If (46) is satisfied, then $\Omega_{M_{1}, M_{2}}^{(\alpha)}$ is a polynomial of degree $\left|\lambda\left(M_{1}\right)\right|+\left|\lambda\left(M_{2}\right)\right|$.

We prove this theorem as follows. First, we show that there is a reduction possible when 0 appears in the encoding of one of the Maya diagrams. Second, we describe the shifting process to reduce $M_{1}$ to $M_{1}+t_{1}$ and $M_{2}$ to $M_{2}+t_{2}$. This is handled in the upcoming two subsections.

Remark 6. In (1), we defined the generalized Laguerre polynomial using the first two types of eigenfunctions of Table 1 . Theorem 1 tells us that if 
we use the four types of eigenfunctions, we can always reduce to the first two types of eigenfunctions.

One could make other choices and shift the Maya diagrams in another way. Then, the generalized Laguerre polynomial is reduced to only two types of eigenfunctions which are not necessarily the first two types as in (1). For example, it is possible to reduce to type one and type three. However, one type of eigenfunctions should be from type one (36) or type four (39), while the other should be from type two (37) or type three (38). The reductions to other combinations can be derived in the same way as Theorem 1.

\subsection{Reduction procedure}

We use the notation

$$
\Omega_{M_{1}, M_{2}}^{(\alpha)}(x)=e^{-\left(r_{2}+r_{4}\right) x} x^{\left(\alpha+r_{1}+r_{2}\right)\left(r_{3}+r_{4}\right)} \cdot \operatorname{Wr}\left[f_{1}, \ldots, f_{r}\right](x)
$$

where $f_{1}, \ldots, f_{r}$ are as in (36)-(39), as before. The following elementary properties of the derivatives will be used in the proof of Lemma 9

$$
\begin{gathered}
\frac{d}{d x}\left(L_{n}^{(\alpha)}(x)\right)=-L_{n-1}^{(\alpha+1)}(x), \\
\frac{d}{d x}\left(e^{x} L_{n}^{(\alpha)}(-x)\right)=e^{x} L_{n}^{(\alpha+1)}(-x), \\
\frac{d}{d x}\left(x^{-\alpha} L_{n}^{(-\alpha)}(x)\right)=(n-\alpha) x^{-\alpha-1} L_{n}^{(-\alpha-1)}(x), \\
\frac{d}{d x}\left(x^{-\alpha} e^{x} L_{n}^{(-\alpha)}(-x)\right)=(n+1) x^{-\alpha-1} e^{x} L_{n+1}^{(-\alpha-1)}(-x) .
\end{gathered}
$$

A reduction is possible if 0 appears in the encoding of one of the Maya diagrams.

LeMmA 9. Let $M_{1}$ and $M_{2}$ be given by (43).

(a) If $n_{r_{1}}=0$, then,

$$
\Omega_{M_{1}, M_{2}}^{(\alpha)}=\left(\prod_{i=1}^{r_{3}}\left(m_{i}^{\prime}-\alpha\right) \prod_{i=1}^{r_{4}}\left(n_{i}^{\prime}+1\right)\right) \Omega_{M_{1}-1, M_{2}}^{(\alpha+1)} .
$$

(b) If $n_{r_{4}}^{\prime}=0$, then,

$$
\Omega_{M_{1}, M_{2}}^{(\alpha)}=(-1)^{r_{1}+r_{2}+r_{4}-1}\left(\prod_{i=1}^{r_{1}}\left(n_{i}+1\right) \prod_{i=1}^{r_{2}}\left(m_{i}+\alpha\right)\right) \Omega_{M_{1}+1, M_{2}}^{(\alpha-1)} .
$$


(c) If $m_{r_{2}}=0$, then,

$$
\Omega_{M_{1}, M_{2}}^{(\alpha)}=(-1)^{r_{2}-1}\left(\prod_{i=1}^{r_{3}}\left(m_{i}^{\prime}+1\right) \prod_{i=1}^{r_{4}}\left(n_{i}^{\prime}-\alpha\right)\right) \Omega_{M_{1}, M_{2}-1}^{(\alpha+1)} .
$$

(d) If $m_{r_{3}}^{\prime}=0$, then,

$$
\Omega_{M_{1}, M_{2}}^{(\alpha)}=(-1)^{r_{1}+r_{2}}\left(\prod_{i=1}^{r_{1}}\left(n_{i}+\alpha\right) \prod_{i=1}^{r_{2}}\left(m_{i}+1\right)\right) \Omega_{M_{1}, M_{2}+1}^{(\alpha-1)} .
$$

Proof. We give the proof of part (b). The other proofs are similar or, in case of part (a), even simpler.

Assume $n_{r_{4}}^{\prime}=0$. Then, $f_{r}(x)=e^{x} x^{-\alpha}$. We take the factor $e^{x} x^{-\alpha}$ out of the Wronskian by means of the identity (12), and we expand the Wronskian determinant along the last row, to obtain

$$
\begin{aligned}
\Omega_{M_{1}, M_{2}}^{(\alpha)}(x)= & \left(e^{x} x^{-\alpha}\right)^{r} e^{-\left(r_{2}+r_{4}\right) x} x^{\left(\alpha+r_{1}+r_{2}\right)\left(r_{3}+r_{4}\right)} \cdot \operatorname{Wr}\left[e^{-x} x^{\alpha} f_{1}, \ldots, e^{-x} x^{\alpha} f_{r-1}, 1\right] \\
= & (-1)^{r-1}\left(e^{x} x^{-\alpha}\right)^{r} e^{-\left(r_{2}+r_{4}\right) x} x^{\left(\alpha+r_{1}+r_{2}\right)\left(r_{3}+r_{4}\right)} \\
& \times \operatorname{Wr}\left[\frac{d}{d x}\left(e^{-x} x^{\alpha} f_{1}\right), \ldots, \frac{d}{d x}\left(e^{-x} x^{\alpha} f_{r-1}\right)\right] .
\end{aligned}
$$

We use the identities

$$
\begin{array}{rlr}
\frac{d}{d x}\left(e^{-x} x^{\alpha} f_{j}\right) & =\frac{d}{d x}\left(e^{-x} x^{\alpha} L_{n_{j}}^{(\alpha)}(x)\right) & \\
& =\left(n_{j}+1\right) x^{\alpha-1} e^{-x} L_{n_{j}+1}^{(\alpha-1)}(x), \quad j=1, \ldots, r_{1}, \\
\frac{d}{d x}\left(e^{-x} x^{\alpha} f_{r_{1}+j}\right) & =\frac{d}{d x}\left(x^{\alpha} L_{m_{j}}^{(\alpha)}(-x)\right) & \\
& =\left(m_{j}+\alpha\right) x^{\alpha-1} L_{m_{j}}^{(\alpha-1)}(-x), \quad j=1, \ldots, r_{2}, \\
\frac{d}{d x}\left(e^{-x} x^{\alpha} f_{r_{1}+r_{2}+j}\right) & =\frac{d}{d x}\left(e^{-x} L_{m_{j}^{\prime}}^{(-\alpha)}(x)\right) & \\
& =-e^{-x} L_{m_{j}^{\prime}}^{(-\alpha+1)}(x), & \\
\frac{d}{d x}\left(e^{-x} x^{\alpha} f_{r_{1}+r_{2}+r_{3}+j}\right) & =\frac{d}{d x}\left(L_{n_{j}^{\prime}}^{(-\alpha)}(-x)\right) & \\
& =L_{n_{j}^{\prime}-1}^{(-\alpha+1)}(-x), &
\end{array}
$$

which follow from (48) to (51). 
Let $\tilde{M}_{1}=M_{1}+1$ and let $\tilde{f}_{1}, \ldots, \tilde{f}_{\tilde{r}}$ be the functions associated with Maya diagram $\tilde{M}_{1}$ and parameter $\tilde{\alpha}=\alpha-1$. We also use $\tilde{r}_{1}=r_{1}, \tilde{r}_{2}=r_{2}$, $\tilde{r}_{3}=r_{3}, \tilde{r}_{4}=r_{4}-1$, and $\tilde{r}=r-1$. The above identities show that

$$
\frac{d}{d x}\left(e^{-x} x^{\alpha} f_{j}\right)=C_{j} e^{-x} x^{\alpha-1} \tilde{f}_{j}, \quad j=1, \ldots, r-1,
$$

where $C_{j}=n_{j}+1$, for $j=1, \ldots, r_{1}, C_{r_{1}+j}=m_{j}+\alpha$, for $j=1, \ldots, r_{2}$, $C_{r_{1}+r_{2}+j}=-1$ for $j=1, \ldots, r_{3}$, and $C_{r_{1}+r_{2}+r_{3}+j}=1$ for $j=1, \ldots, r_{4}-1$.

We take the constant factor $C_{j}$ out of the $j$ th column of the Wronskian, for each $j=1, \ldots, r$, and then take out the common factor $e^{-x} x^{\alpha-1}$ by means of the identity (12). This leads to a prefactor $\left(e^{-x} x^{\alpha-1}\right)^{r-1}$ and thus

$$
\begin{aligned}
\Omega_{M_{1}, M_{2}}^{(\alpha)}(x)= & c_{1}\left(e^{x} x^{-\alpha}\right)^{r}\left(e^{-x} x^{\alpha-1}\right)^{r-1} e^{-\left(r_{2}+r_{4}\right) x} x^{\left(\alpha+r_{1}+r_{2}\right)\left(r_{3}+r_{4}\right)} \\
& \times \operatorname{Wr}\left[\tilde{f}_{1}, \ldots, \tilde{f}_{r-1}\right] \\
= & c_{1} e^{-\left(r_{2}+r_{4}-1\right) x} x^{\left(\alpha+r_{1}+r_{2}\right)\left(r_{3}+r_{4}\right)-r+1} \cdot \operatorname{Wr}\left[\tilde{f}_{1}, \ldots, \tilde{f}_{r-1}\right]
\end{aligned}
$$

where

$$
c_{1}=(-1)^{r-1} \prod_{j=1}^{r_{4}-1} C_{j}=(-1)^{r_{1}+r_{2}+r_{4}-1} \prod_{j=1}^{r_{1}}\left(n_{j}+1\right) \prod_{j=1}^{r_{2}}\left(m_{j}+\alpha\right) .
$$

Hence, (53) follows because

$$
\begin{aligned}
\left(\alpha+r_{1}+r_{2}\right)\left(r_{3}+r_{4}\right)-r+1 & =\left(\alpha-1+r_{1}+r_{2}\right)\left(r_{3}+r_{4}-1\right) \\
& =\left(\tilde{\alpha}+\tilde{r}_{1}+\tilde{r}_{2}\right)\left(\tilde{r}_{3}+\tilde{r}_{4}\right) .
\end{aligned}
$$

\subsection{Shifting process}

First, assume that $t_{1} \leq 0$ and $t_{2} \leq 0$. Then, $r_{3}=r_{4}=0, r_{1} \geq\left|t_{1}\right|-1$ with $n_{r_{1}-j}=j$, for $j=0, \ldots,\left|t_{1}\right|-1$ and $r_{2} \geq\left|t_{2}\right|-1$ with $m_{r_{2}-j}=j$, for $j=0, \ldots,\left|t_{2}\right|-1$. Therefore, we can apply (52) $\left|t_{1}\right|$ times and (55) $\left|t_{2}\right|$ times. We find that

$$
\Omega_{M_{1}, M_{2}}^{(\alpha)}=C(\alpha) \Omega_{M_{1}+t_{1}, M_{2}+t_{2}}^{\left(\alpha-t_{1}-t_{2}\right)},
$$

where

$$
C(\alpha)=(-1)^{\sum_{i=1}^{\left|t_{2}\right|} r_{2}-i}=(-1)^{d_{0}} .
$$

Second, assume that $t_{1}>0$ and denote

$$
\tilde{M}_{1}=M_{1}+1: \quad\left(\tilde{n}_{1}^{\prime}, \ldots, \tilde{n}_{\tilde{r}_{4}}^{\prime} \mid \tilde{n}_{1}, \ldots, \tilde{n}_{\tilde{r}_{1}}\right) .
$$

There are two situations, depending on whether the box immediately to the left of the origin in $M_{1}$ is filled or not. 
- If it is empty, then $n_{r_{4}}^{\prime}=0, \tilde{r}_{4}=r_{4}-1, \tilde{r}_{1}=r_{1}$, and

$$
\tilde{M}_{1}: \quad\left(n_{1}^{\prime}-1, \ldots, n_{r_{4}-1}^{\prime}-1 \mid n_{1}+1, \ldots, n_{r_{1}}+1\right) .
$$

- If it is filled, then $\tilde{n}_{r_{1}+1}=0, \tilde{r}_{4}=r_{4} \tilde{r}_{1}=r_{1}+1$, and

$$
\tilde{M}_{1}: \quad\left(n_{1}^{\prime}-1, \ldots, n_{r_{4}}^{\prime}-1 \mid n_{1}+1, \ldots, n_{r_{1}}+1,0\right) .
$$

In both cases, a reduction procedure is possible, because either $n_{r_{4}}^{\prime}=0$ or $\tilde{n}_{r_{1}+1}=0$. If $n_{r_{4}}^{\prime}=0$, we use (53) and if $\tilde{n}_{r_{1}+1}=0$, we use (52) but with $M_{1}+1$ instead of $M_{1}$ and $\alpha-1$ instead of $\alpha$, i.e.,

$$
\Omega_{M_{1}, M_{2}}^{(\alpha)}=\left(\prod_{i=1}^{r_{3}}\left(m_{i}^{\prime}-\alpha+1\right) \prod_{i=1}^{r_{4}} n_{i}^{\prime}\right)^{-1} \Omega_{M_{1}+1, M_{2}}^{(\alpha-1)}
$$

Continuing in this way, we go from $M_{1}$ to $M_{1}+t_{1}$, to arrive at a Maya diagram with no empty boxes on the left. We find

$$
\Omega_{M_{1}, M_{2}}^{(\alpha)}=C_{1}(\alpha) \Omega_{M_{1}+t_{1}, M_{2}}^{\left(\alpha-t_{1}\right)}
$$

for some constant $C_{1}(\alpha)$. Now, we perform a second step where we shift $M_{2}$ to $M_{2}+t_{2}$. Hence, in a similar way, we find for some constant $C_{2}(\alpha)$

$$
\Omega_{M_{1}+t_{1}, M_{2}}^{\left(\alpha-t_{1}\right)}=C_{2}(\alpha) \Omega_{M_{1}+t_{1}, M_{2}+t_{2}}^{\left(\alpha-t_{1}-t_{2}\right)}
$$

Hence,

$$
\Omega_{M_{1}, M_{2}}^{(\alpha)}=C_{1}(\alpha) C_{2}(\alpha) \Omega_{M_{1}+t_{1}, M_{2}+t_{2}}^{\left(\alpha-t_{1}-t_{2}\right)}
$$

which means in terms of the partitions $\lambda$ and $\mu$ that indeed

$$
\Omega_{M_{1}, M_{2}}^{(\alpha)}=C(\alpha) \Omega_{\lambda, \mu}^{\left(\alpha-t_{1}-t_{2}\right)}
$$

where $C(\alpha)=C_{1}(\alpha) C_{2}(\alpha)$.

Third, when $t_{2}>0$, we first shift the Maya diagram $M_{2}$ to $M_{2}+t_{2}$. Next, we transpose $M_{1}$ to $M_{1}+t_{1}$ and we find that 60 also holds.

The constant $C(\alpha)$ can be found by keeping track of all the prefactors we find along the way. There is a fair amount of cancellation taking place. In particular, every factor that we would find in the denominator also appears somewhere in a numerator. It leads to the formula (44). However, knowing (44), we can also verify it by induction on the value of $T=\left|t_{1}\right|+\left|t_{2}\right|$.

If $t_{1}=t_{2}=0$, then $r_{3}=r_{4}=0$, and all products in (44) are empty and $d_{0}=0$. We then find $C(\alpha)=1$ as it should be.

Take $T>0$ and assume the formula (44) is correct whenever $\left|t_{1}\right|+\left|t_{2}\right|=$ $T-1$. Assuming $\left|t_{1}\right|+\left|t_{2}\right|=T$, we have a number of cases to consider. Note that we may assume that $t_{1}>0$ or $t_{2}>0$ as the situation where $t_{1}, t_{2} \leq 0$ is derived in (56) which also covers $t_{1}=t_{2}=0$.

(a) $t_{1}>0$ and $\tilde{M}_{1}=M_{1}+1$ is given by $(57)$, 
(b) $t_{1}>0$ and $\tilde{M}_{1}=M_{1}+1$ is given by (58),

(c) $t_{2}>0$ and $\tilde{M}_{2}=M_{1}+1$ is similar as (57),

(d) $t_{2}>0$ and $\tilde{M}_{2}=M_{1}+1$ is similar as (58).

As the ideas are similar for each case, we only treat case (b). In that case, we have (59) and thus

$$
C\left(\alpha, M_{1}, M_{2}\right)=C\left(\alpha-1, M_{1}+1, M_{2}\right)\left(\prod_{i=1}^{r_{3}}\left(m_{i}^{\prime}-\alpha+1\right) \prod_{i=1}^{r_{4}} n_{i}^{\prime}\right)^{-1} .
$$

By the induction hypothesis,

$$
\begin{aligned}
C\left(\alpha-1, M_{1}+1, M_{2}\right)= & (-1)^{\tilde{d}} \prod_{j=1}^{\tilde{r}_{1}} \prod_{k=1}^{r_{3}}\left(m_{k}^{\prime}-(\alpha-1)-\tilde{n}_{j}\right) \\
& \times \prod_{j=1}^{r_{2}} \prod_{k=1}^{\tilde{r}_{4}}\left(\tilde{n}_{k}^{\prime}-(\alpha-1)-m_{j}\right) \prod_{j=1}^{\tilde{r}_{1}} \prod_{k=1}^{r_{4}}\left(\tilde{n}_{j}+\tilde{n}_{k}^{\prime}+1\right) \\
& \times \prod_{j=1}^{r_{2}} \prod_{k=1}^{r_{3}}\left(m_{j}+m_{k}^{\prime}+1\right)
\end{aligned}
$$

with the parameters $\tilde{r}_{1}=r_{1}+1, \tilde{r}_{4}=r_{4}, \tilde{n}_{j}=n_{j}+1$ for $j=1, \ldots, r_{1}$, $\tilde{n}_{r_{1}+1}=0$, and $\tilde{n}_{j}^{\prime}=n_{j}^{\prime}-1$ for $j=1, \ldots, r_{4}$, as they are associated with $\tilde{M}_{1}$, see (58). The first three double products appearing in (62) are

$$
\begin{aligned}
\prod_{j=1}^{\tilde{r}_{1}} \prod_{k=1}^{r_{3}}\left(m_{k}^{\prime}-(\alpha-1)-\tilde{n}_{j}\right) & =\prod_{j=1}^{r_{1}} \prod_{k=1}^{r_{3}}\left(m_{k}^{\prime}-\alpha-n_{j}\right) \prod_{k=1}^{r_{3}}\left(m_{k}^{\prime}-(\alpha-1)\right), \\
\prod_{j=1}^{r_{2}} \prod_{k=1}^{\tilde{r}_{4}}\left(\tilde{n}_{k}^{\prime}-(\alpha-1)-m_{j}\right) & =\prod_{j=1}^{r_{2}} \prod_{k=1}^{r_{4}}\left(n_{k}^{\prime}-\alpha-m_{j}\right), \\
\prod_{j=1}^{\tilde{r}_{1}} \prod_{k=1}^{\tilde{r}_{4}}\left(\tilde{n}_{j}+\tilde{n}_{k}^{\prime}+1\right) & =\prod_{j=1}^{r_{1}} \prod_{k=1}^{r_{4}}\left(n_{j}+n_{k}^{\prime}+1\right) \prod_{k=1}^{r_{4}} n_{k}^{\prime} .
\end{aligned}
$$

Combining this with (61) and (62), we obtain (44) up to the sign. So far, we only have to proof that $(-1)^{\tilde{d}}=(-1)^{d}$. The number $\tilde{d}$ depends on $\tilde{M}_{1}$ and $M_{2}$ where $d$ depends on $M_{1}$ and $M_{2}$. Trivially, $\tilde{d}_{0}=d_{0}$ and $\tilde{d}_{2}=d_{2}$ as they are independent of the first Maya diagram. The induction hypothesis gives us

$$
\tilde{d}_{1}=\frac{\tilde{r}_{4}\left(\tilde{r}_{4}-1\right)}{2}+\tilde{r}_{1} \tilde{r}_{4}+\frac{\left(\tilde{n}_{1}^{\prime}+1-\tilde{r}_{4}\right)\left(\tilde{n}_{1}^{\prime}+2+\tilde{r}_{4}\right)}{2}+\sum_{i=1}^{\tilde{n}_{1}^{\prime}+1-\tilde{r}_{4}} \tilde{n}_{\tilde{r}_{1}+i}
$$




$$
=\frac{r_{4}\left(r_{4}-1\right)}{2}+\left(r_{1}+1\right) r_{4}+\frac{\left(n_{1}^{\prime}-r_{4}\right)\left(n_{1}^{\prime}+1+r_{4}\right)}{2}+\sum_{i=1}^{n_{1}^{\prime}-r_{4}}\left(n_{r_{1}+1+i}+1\right) .
$$

The box to the left of the origin of $M_{1}$ is filled and hence $n_{r_{1}+1}=-1$. Therefore,

$$
\begin{aligned}
\tilde{d}_{1}= & \frac{r_{4}\left(r_{4}-1\right)}{2}+r_{1} r_{4}+r_{4}+\frac{\left(n_{1}^{\prime}+1-r_{4}\right)\left(n_{1}^{\prime}+2+r_{4}\right)}{2}-n_{1}^{\prime}-1 \\
& +n_{1}^{\prime}-r_{4}+\sum_{i=1}^{n_{1}^{\prime}-r_{4}} n_{r_{1}+1+i} \\
= & \frac{r_{4}\left(r_{4}-1\right)}{2}+r_{1} r_{4}+\frac{\left(n_{1}^{\prime}+1-r_{4}\right)\left(n_{1}^{\prime}+2+r_{4}\right)}{2}+\sum_{i=1}^{n_{1}^{\prime}+1-r_{4}} n_{r_{1}+i} .
\end{aligned}
$$

Hence, $\tilde{d}_{1}=d_{1}$ such that $(-1)^{\tilde{d}}=(-1)^{d}$. This ends the proof of Theorem 1 .

\subsection{Proof of Lemma 5}

From the previous subsections, it should be clear that we can play with the Maya diagrams to obtain several results. For example, Lemma 5 can be viewed as a special case of Theorem 1.

Proof of Lemma 5. Take the following Maya diagrams,

$$
\begin{array}{ll}
M_{1}: & \left(n_{1}^{\prime}, \ldots, n_{r_{4}}^{\prime} \mid \varnothing\right), \\
M_{2}: & \left(m_{1}^{\prime}, \ldots, m_{r_{3}}^{\prime} \mid \varnothing\right),
\end{array}
$$

where $n_{r_{4}}^{\prime} \neq 0$ if $r_{4} \geq 1$ and $m_{r_{3}}^{\prime} \neq 0$ if $r_{3} \geq 0$. In this situation, we have that

$$
\Omega_{M_{1}, M_{2}}^{(\alpha)}(x)=e^{-r_{4} x} x^{\alpha\left(r_{3}+r_{4}\right)} \cdot \operatorname{Wr}\left[f_{1}, \ldots, f_{r}\right]
$$

where $r=r_{3}+r_{4}$ and $f_{1}, \ldots, f_{r}$ are as in (38)and (39) with $r_{1}=r_{2}=0$. Using (12) with $g(x)=x^{-\alpha}$, and comparing with (7), we get that

$$
\Omega_{M_{1}, M_{2}}^{(\alpha)}=\Omega_{\mu^{\prime}, \lambda^{\prime}}^{(-\alpha)}
$$

where $\mu^{\prime}$ and $\lambda^{\prime}$ are the conjugate partitions. We apply Theorem 1 to the left-hand side, where we note that all double products are empty because $r_{1}=r_{2}=0$, and at the same time use Lemma 3 to interchange the two partitions in the right-hand side of (64). We end up with

$$
(-1)^{d_{1}+d_{2}} \Omega_{\lambda, \mu}^{(\alpha-t)}(x)=(-1)^{\frac{r_{3}\left(r_{3}-1\right)}{2}+\frac{r_{4}\left(r_{4}-1\right)}{2}} \Omega_{\lambda^{\prime}, \mu^{\prime}}^{(-\alpha)}(-x)
$$


In this special situation, we have

$$
\begin{aligned}
& d_{1}=|\lambda|+\frac{r_{4}\left(r_{4}-1\right)}{2}, \\
& d_{2}=|\mu|+\frac{\left(m_{1}^{\prime}+1-r_{3}\right)\left(m_{1}^{\prime}-r_{3}\right)}{2} .
\end{aligned}
$$

The value $m_{1}^{\prime}+1-r_{3}$ is the number of empty boxes to the left of the origin of $M_{2}$ and therefore it is equal to the length of the partition $\mu$, which we denote here by $r(\mu)$. Similarly, we have $r(\lambda)$ as the length of the partition $\lambda$. We also note that $\mu_{1}=r_{3}$, and then (65) reduces to

$$
\Omega_{\lambda, \mu}^{(\alpha-t)}(x)=(-1)^{\frac{\mu_{1}\left(\mu_{1}-1\right)}{2}+|\lambda|+|\mu|+\frac{r(\mu)(r(\mu)-1)}{2}} \Omega_{\lambda^{\prime}, \mu^{\prime}}^{(-\alpha)}(-x) .
$$

This is the result of Lemma 5 if we replace $\alpha$ by $\alpha+t$.

However, it remains to check that the value of $t$ used in Lemma 5 agrees with the one from Theorem 1 for the special case (63). In Theorem 1, we have $t=t_{1}+t_{2}$ with $t_{1}=t\left(M_{1}\right)$ and $t_{2}=t\left(M_{2}\right)$. For the Maya diagrams (63), this is $t_{1}=n_{1}^{\prime}+1$ and $t_{2}=m_{1}^{\prime}+1$. In Lemma 5, we have $t=\lambda_{1}+\mu_{1}+r(\lambda)+r(\mu)$. And indeed, it holds true that $\lambda_{1}+r(\lambda)=n_{1}^{\prime}+1$ and $\mu_{1}+r(\mu)=m_{1}^{\prime}+1$. This completes the proof of the lemma.

\section{Construction of the exceptional Laguerre polynomial}

Exceptional Laguerre polynomials are often denoted as $X_{m}$-Laguerre polynomials. In this section, we prove that the exceptional Laguerre polynomial defined in (17) using two partitions captures the most general format and we give explicit identities with respect to the definitions of $X_{m}$-Laguerre polynomials.

\subsection{Exceptional Laguerre polynomial revisited}

The exceptional Laguerre polynomial is obtained by adding one eigenfunction to the Wronskian of the generalized Laguerre polynomial. We defined two kinds of exceptional Laguerre polynomials in (17) and (18). They are related by (19) and therefore we only considered (17). In general, we could take $f_{1}, \ldots, f_{r}$ as in (36)-(39) and add one more function into the Wronskian where this function is one of the four types. By the reduction procedure, we can always reduce each set up to definition (17) as stated in the following proposition. Therefore, our definition of the exceptional Laguerre polynomial (17) covered all possibilities. Recall that $\lambda^{\prime}$ is the conjugated partition of $\lambda$. 
Proposition 3. Let $f_{1}, \ldots, f_{r}$ as in (36)-(39) and take two Maya diagrams $M_{1}, M_{2}$ as in (43). Let $\lambda=\lambda\left(M_{1}\right)$ and $\mu=\lambda\left(M_{2}\right)$ be the partitions that are associated with these Maya diagrams and let $r(\lambda)$ and $r(\mu)$ denote the length of these partitions. Let $t_{1}=t\left(M_{1}\right), t_{2}=t\left(M_{2}\right)$ as in (42). Take $s \geq 0$.

(a) If $s \neq n_{i}$ for $i=1, \ldots, r_{1}$, then $n:=s+t_{1}+|\lambda|+|\mu|-r(\lambda) \in \mathbb{N}_{\lambda, \mu}$ and

$$
e^{-\left(r_{2}+r_{4}\right) x} x^{\left(\alpha+r_{1}+1+r_{2}\right)\left(r_{3}+r_{4}\right)} \cdot \operatorname{Wr}\left[f_{1}, \ldots, f_{r}, L_{s}^{(\alpha)}(x)\right](x)=C_{1} L_{\lambda, \mu, n}^{\left(\alpha-t_{1}-t_{2}\right)}(x),
$$

for some constant $C_{1}$.

(b) If $s \neq m_{i}$ for $i=1, \ldots, r_{2}$, then $n:=s+t_{2}+|\lambda|+|\mu|-r(\mu) \in$ $\mathbb{N}_{\mu, \lambda}$ and

$$
\begin{aligned}
& e^{-\left(r_{2}+1+r_{4}\right) x} x^{\left(\alpha+r_{1}+r_{2}+1\right)\left(r_{3}+r_{4}\right)} \cdot \operatorname{Wr}\left[f_{1}, \ldots, f_{r}, e^{x} L_{s}^{(\alpha)}(-x)\right](x) \\
& \quad=C_{2} L_{\mu, \lambda, n}^{\left(\alpha-t_{1}-t_{2}\right)}(-x)
\end{aligned}
$$

for some constant $C_{2}$.

(c) If $s \neq m_{i}^{\prime}$ for $i=1, \ldots, r_{3}$ then $n:=s+m_{1}+1+|\lambda|+|\mu|-$ $r\left(\mu^{\prime}\right) \in \mathbb{N}_{\mu^{\prime}, \lambda^{\prime}}$ and

$$
\begin{aligned}
& e^{-\left(r_{2}+r_{4}\right) x} x^{\left(\alpha+r_{1}+r_{2}\right)\left(r_{3}+1+r_{4}\right)} \cdot \operatorname{Wr}\left[f_{1}, \ldots, f_{r}, x^{-\alpha} L_{s}^{(-\alpha)}(x)\right](x) \\
& \quad=C_{3} L_{\mu \prime, \lambda \prime, n}^{(-\alpha \prime)}(x),
\end{aligned}
$$

where $\alpha^{\prime}=\alpha+n_{1}+m_{1}+2$ and for some constant $C_{3}$.

(d) If $s \neq n_{i}^{\prime}$ for $i=1, \ldots, r_{4}$ then $n:=s+n_{1}+1+|\lambda|+|\mu|-r\left(\lambda^{\prime}\right) \in$ $\mathbb{N}_{\lambda^{\prime}, \mu^{\prime}}$, then

$$
\begin{aligned}
& e^{-\left(r_{2}+r_{4}+1\right) x} x^{\left(\alpha+r_{1}+r_{2}\right)\left(r_{3}+r_{4}+1\right)} \cdot \operatorname{Wr}\left[f_{1}, \ldots, f_{r}, e^{x} x^{-\alpha} L_{s}^{(-\alpha)}(x)\right](x) \\
& \quad=C_{4} L_{\lambda^{\prime}, \mu^{\prime}, n}^{\left(-\alpha^{\prime}\right)}(-x),
\end{aligned}
$$

where $\alpha^{\prime}=\alpha+n_{1}+m_{1}+2$ and for some constant $C_{4}$.

Proof.

(a) By Theorem 1, we can shift the origin in both Maya diagrams to its canonical choice corresponding to both partitions $\lambda$ and $\mu$. By this process, $L_{s}^{(\alpha)}(x)$ shifts to $L_{s+t_{1}}^{\left(\alpha-t_{1}-t_{2}\right)}(x)$. Therefore, we end up with definition (17) and the degree is given by $n=s+t_{1}+|\lambda|+$ $|\mu|-r(\lambda) \in \mathbb{N}_{\lambda, \mu}$. Note that the two conditions of $n \in \mathbb{N}_{\lambda, \mu}$ reduce to $s+t_{1} \geq 0$ and $s \neq n_{i}$ for $i=1, \ldots, r_{1}$. The second condition is fulfilled by assumption and the first condition is trivially true by construction. 
(b) We apply the same procedure as in (a). In this case, the last function in the Wronskian $e^{x} L_{s}^{(\alpha)}(-x)$ shifts to $e^{x} L_{s+t_{2}}^{\left(\alpha-t_{1}-t_{2}\right)}(-x)$. Hence, we end up with definition (18) with degree $s+t_{2}+|\lambda|+|\mu|-r(\mu) \epsilon$ $\mathbb{N}_{\mu, \lambda}$. By (19), interchanging the partitions gives definition (17) up to a possible sign change.

(c) We shift both origins of the Maya diagrams to the right in such a way that all boxes to the right are empty, and the first box to the left is filled: $n_{1}+1$ steps for the first Maya diagram and $m_{1}+1$ steps for the second. This choice is related to the conjugated partition. Set $\alpha^{\prime}=\alpha+n_{1}+m_{1}+2$. Similar as in Theorem 1, up to a constant, we arrive at

$$
e^{-\left(r\left(\lambda^{\prime}\right)+1\right) x} x^{\alpha^{\prime}\left(r\left(\lambda^{\prime}\right)+r\left(\mu^{\prime}\right)+1\right)} \cdot \operatorname{Wr}\left[g_{1}, \ldots, g_{r\left(\lambda^{\prime}\right)}, g_{r\left(\lambda^{\prime}\right)+1}, \ldots, g_{r\left(\lambda^{\prime}\right)+r\left(\mu^{\prime}\right)}, g\right]
$$

where

$$
\begin{array}{ll}
g_{j}(x)=e^{x} x^{-\alpha^{\prime}} L_{\bar{n}_{j}}^{\left(-\alpha^{\prime}\right)}(-x), & j=1, \ldots, r\left(\lambda^{\prime}\right), \\
g_{r\left(\lambda^{\prime}\right)+j}=x^{-\alpha^{\prime}} L_{\bar{m}_{j}}^{\left(-\alpha^{\prime}\right)}(x), & j=1, \ldots, r\left(\mu^{\prime}\right)
\end{array}
$$

for some nonnegative integers $\bar{n}_{j}, \bar{m}_{j}$ and

$$
g(x)=e^{x} x^{-\alpha^{\prime}} L_{s+m_{1}+1}^{\left(-\alpha^{\prime}\right)}(x)
$$

because the function $e^{x} x^{-\alpha} L_{s}^{(-\alpha)}(x)$ is shifted to $e^{x} x^{-\alpha^{\prime}} L_{s+m_{1}+1}^{\left(-\alpha^{\prime}\right)}(x)$. Hence, all functions in the Wronskian consist of a common factor $x^{-\alpha^{\prime}}$ which cancels out by the factor in front of the Wronskian by (12). Next, we can interchange the functions $g_{j}$ such that we end up with definition (17) where the degree is $s-m_{1}-1+\left|\lambda^{\prime}\right|+\left|\mu^{\prime}\right|-r\left(\mu^{\prime}\right)$ and the parameter is $-\alpha^{\prime}$. The identity follows as $\left|\lambda^{\prime}\right|=|\lambda|$.

(d) The steps are similar to (c); however, we end up with definition (18). By (19), the result follows.

\section{2. $X_{m}$-Laguerre polynomials}

In the literature, exceptional Laguerre polynomials are often denoted as $X_{m}$-Laguerre polynomials where $m$ is the number of exceptional degrees [3-8, 10, 11,21-23,27-29]. In our set-up, this number of exceptional degrees is given by

$$
m=\left|\mathbb{N}_{0} \backslash \mathbb{N}_{\lambda, \mu}\right|=|\lambda|+|\mu|,
$$

where $\mathbb{N}_{\lambda, \mu}$ is the degree sequence (16). Hence, to obtain exactly $m$ exceptional degrees, we have to take the partitions $\lambda$ and $\mu$ in such a way that (67) is satisfied. A few of these possibilities are studied in detail and referred as type I, type II, and type III exceptional Laguerre polynomials. 
In [7], these polynomials are denoted by $L_{m, n}^{I, \alpha}, L_{m, n}^{I I, \alpha}, L_{m, n}^{I I I, \alpha}$ where $m$ is the number of exceptional degrees, $n$ is the degree of the polynomial, $\alpha$ is a parameter, and the Roman number relates to the corresponding type. The definitions of these polynomials are given below. As these three types only cover a small part of all exceptional Laguerre polynomials, we do not prefer this terminology and notation. The approach with partitions is more general and covers all cases, as we have shown.

We show how the $L_{m, n}^{I, \alpha}, L_{m, n}^{I I, \alpha}, L_{m, n}^{I I I, \alpha}$ exceptional Laguerre polynomials are expressed in terms of partitions.

Proposition 4. Let $m \geq 0$ be fixed. We have the following identities,

$$
\begin{array}{cc}
L_{m, n}^{I, \alpha}=-L_{\emptyset,(m), n}^{(\alpha-1)}, & n \geq m, \\
L_{m, n}^{I I, \alpha}=(-1)^{\frac{m(m+3)}{2}(2 m-n-\alpha-1) \cdot L_{\emptyset,(1, \ldots, 1), n}^{(\alpha-m)},} & n \geq m, \\
L_{m, n}^{I I I, \alpha}=-n \cdot L_{(1, \ldots, 1), \emptyset, n}^{(\alpha-m)} & n>m .
\end{array}
$$

The partition $(1, \ldots, 1)$ in (69) and (70) consists of $m$ values 1 . Its Young diagram consists of one single column of length $m$. The partition $(m)$ in (68) is its conjugate partition, and its Young diagram consists of one row of length $m$.

The identities of Proposition 4 are derived below, see (71), (75), and (77), and were verified with Maple for the examples given in the appendices of $[7,27]$. For the remaining of this subsection, let $m$ be fixed.

Type I exceptional Laguerre polynomial. The polynomial is given by

$$
L_{m, n}^{I, \alpha}(x):=\left|\begin{array}{cc}
L_{m}^{(\alpha)}(-x) & -L_{n-m-1}^{(\alpha)}(x) \\
L_{m}^{(\alpha-1)}(-x) & L_{n-m}^{(\alpha-1)}(x)
\end{array}\right|
$$

for $n \geq m$, see [7, Formula (3.2)]. If we interchange the rows in this determinant and use the derivatives (48) and (49), we obtain that

$$
L_{m, n}^{I, \alpha}=-e^{-x} \cdot \operatorname{Wr}\left[f_{1}, L_{n-m}^{(\alpha-1)}\right],
$$

where

$$
f_{1}(x)=e^{x} L_{m}^{(\alpha-1)}(-x)
$$

Hence, by definition (17),

$$
L_{m, n}^{I, \alpha}=-L_{\emptyset,(m), n}^{(\alpha-1)},
$$

where $\lambda=\emptyset$ and $\mu=(m)$. By Lemma 6 , these type I $X_{m}$-Laguerre polynomials form a complete set on the positive real line with respect 
to the measure $\frac{x^{\alpha} e^{-x}}{\left(\Omega_{\emptyset,(m)}^{(\alpha-1)}\right)^{2}}$ if $\alpha-1>-1$. Moreover, the generalized Laguerre polynomial is related to a classical Laguerre polynomial, i.e.,

$$
\Omega_{\emptyset,(m)}^{(\alpha-1)}(x)=L_{m}^{(\alpha-1)}(-x)
$$

for all $x \in \mathbb{C}$ which follows directly by definition (7).

Type II exceptional Laguerre polynomial. This polynomial is defined as

$$
L_{m, n}^{I I, \alpha}(x):=\left|\begin{array}{cc}
x L_{m}^{(-\alpha-1)}(x) & -L_{n-m}^{(\alpha+1)}(x) \\
(m-\alpha-1) L_{m}^{(-\alpha-2)}(x) & L_{n-m-1}^{(\alpha+2)}(x)
\end{array}\right|
$$

for $n \geq m$, see [7, Section 4]. If we use (48) and (50), we can rewrite this polynomial as

$$
L_{m, n}^{I I, \alpha}=-x^{\alpha+2} \cdot \operatorname{Wr}\left[f_{1}, f_{2}\right]
$$

where

$$
\begin{aligned}
& f_{1}(x)=x^{-\alpha-1} L_{m}^{(-\alpha-1)}(x), \\
& f_{2}(x)=L_{n-m}^{(\alpha+1)}(x) .
\end{aligned}
$$

The eigenfunction $f_{2}$ corresponds to (36) and $f_{1}$ to (38). Now the idea is that we apply Theorem 1 to transfer the eigenfunction $f_{2}$ to a set of eigenfunctions related to (37). In fact, we transpose the second Maya diagram to its canonical choice $(41)$, i.e., $M_{2}:(m \mid \emptyset) \rightarrow(\emptyset \mid m, m-1, \ldots, 1)$. This process is obtained by shifting the origin $m+1$ steps to the left and therefore the parameter $\alpha+1$ decreases by $m+1$. We also get extra factors while doing these steps. Encode the first Maya diagram $M_{1}:(\emptyset \mid n-m)$ which is already in the canonical choice, we get that

$$
\begin{aligned}
L_{m, n}^{I I, \alpha} & =\Omega_{M_{1}, M_{2}}^{(\alpha+1)} \\
& =(-1)^{\frac{m(m+1)}{2}}(2 m-n-\alpha-1) \cdot \Omega_{\lambda\left(M_{1}\right), \lambda\left(M_{2}\right)}^{(\alpha-m)},
\end{aligned}
$$

where the first equality follows by interchanging $f_{1}$ and $f_{2}$ in (72) and the second equality is obtained by applying Theorem 1. As a final step, we want the first function in the Wronskian of $\Omega_{\lambda\left(M_{1}\right), \lambda\left(M_{2}\right)}^{(\alpha+1)}$ to be the last eigenfunction such that we obtain the same order as in definition (17). Therefore, we interchange the functions in the Wronskian which leads to an extra factor $(-1)^{m}$, i.e.,

$$
\Omega_{\lambda\left(M_{1}\right), \lambda\left(M_{2}\right)}^{(\alpha-m)}=(-1)^{m} \cdot L_{\emptyset,(1, \ldots, 1), n}^{(\alpha-m)},
$$


where the length of the partition $\mu$ is given by $m$ such that $r=m$. If we combine (73) and (74), we end up with

$$
L_{m, n}^{I I, \alpha}=(-1)^{\frac{m(m+3)}{2}}(2 m-n-\alpha-1) \cdot L_{\emptyset,(1, \ldots, 1), n}^{(\alpha-m)} .
$$

Hence, by Lemma 6 , these type II $X_{m}$-Laguerre polynomials form an orthogonal complete set on the positive real line with respect to the measure $\frac{x^{\alpha} e^{-x}}{\left(\Omega_{\emptyset,(1, \ldots, 1)}^{(\alpha-m)}\right)^{2}}$ if $\alpha-m>-1$. The generalized Laguerre polynomial can be written as a Laguerre polynomial, i.e.,

$$
\Omega_{\emptyset,(1, \ldots, 1)}^{(\alpha-m)} \equiv(-1)^{\frac{m(m+1)}{2}} L_{m}^{(-\alpha-1)}
$$

which follows by Theorem 1 .

Type III exceptional Laguerre polynomial. The type III exceptional Laguerre polynomial is obtained in [7, Section 5] and is defined as

$$
L_{m, n}^{I I I, \alpha}(x):=\left|\begin{array}{cc}
x L_{m}^{(-\alpha-1)}(-x) & -L_{n-m-1}^{(\alpha+1)}(x) \\
(m+1) L_{m}^{(-\alpha-2)}(-x) & L_{n-m-2}^{(\alpha+2)}(x)
\end{array}\right|
$$

for $n>m$, while for $n=0$ this polynomial is defined as the constant function 1 . We can use the derivatives (48) and (51) to obtain

$$
L_{m, n}^{I I I, \alpha}=-x^{\alpha+2} e^{-x} \cdot \operatorname{Wr}\left[f_{1}, f_{2}\right],
$$

for $n>m$ and where

$$
\begin{aligned}
& f_{1}(x)=x^{-\alpha-1} e^{x} L_{m}^{(-\alpha-1)}(-x), \\
& f_{2}(x)=L_{n-m-1}^{(\alpha+1)}(x) .
\end{aligned}
$$

The function $f_{1}$ corresponds to (39). Similar as in the type II case, we shift the (first) Maya diagram to its canonical form by Theorem 1 , i.e., $M_{1}:(m \mid n-m-1) \rightarrow(\emptyset \mid n, m, m-1, \ldots, 1)$. Hence, we end up with only eigenfunctions of category (36). As a last step, we transpose the first eigenfunction to the end of the Wronskian such that the order of the degrees is the same as in definition $(17)$, i.e. $(n, m, m-1, \ldots, 1) \rightarrow$ $(m, m-1, \ldots, 1, n)$. Combining all extra factors, we obtain the identity

$$
L_{m, n}^{I I I, \alpha}=-n \cdot L_{(1, \ldots, 1), \emptyset, n}^{(\alpha-m)}
$$

for $n>m$ and where the length of the partition $\lambda$ is given by $m$ such that $r=m$. Lemma 6 tells us that these type III $X_{m}$-Laguerre polynomials form an orthogonal complete set to the measure $\frac{x^{\alpha} e^{-x}}{\left(\Omega_{(1, \ldots, 1), \emptyset}^{(\alpha-m)}\right.}$ if $m$ is even and $\alpha-m>-1$. The generalized Laguerre polynomial can be written as a Laguerre polynomial, i.e.,

$$
\Omega_{(1, \ldots, 1), \emptyset}^{(\alpha-m)}(x)=(-1)^{m} L_{m}^{(-\alpha-1)}(-x)
$$


for all $x \in \mathbb{C}$ which follows by Theorem 1 or by applying identity (11) on (76).

\section{Zeros of exceptional Laguerre polynomials: new results}

In this section, we state our new results according to the zeros of exceptional Laguerre polynomials. From now on, we assume that $\alpha \in \mathbb{R}$ and the two partitions $\lambda$ and $\mu$ are fixed. As before, the length of the partition $\lambda$ is $r_{1}$ and for $\mu$ it is $r_{2}$. Let $r=r_{1}+r_{2}$. Throughout, we also use $\left(n_{1}, \ldots, n_{r_{1}}\right)$ and $\left(m_{1}, \ldots, m_{r_{2}}\right)$ as in (6). Our definition of exceptional Laguerre polynomials generalizes the $X_{m}$-Laguerre polynomials considered in $[7,28]$ and our results on zeros are generalizations of some of the results in these papers, see [28, Sections 3 and 4] and [7, Section 5.5].

\subsection{Number of positive real zeros}

Under the conditions of Lemma 6, the polynomials are a complete orthogonal system, and they are also eigenfunctions for a Sturm-Liouville problem on $[0, \infty)$. From general Sturm-Liouville theory, see, for example [36], the number of positive real zeros of $L_{\lambda, \mu, n}^{(\alpha)}$ is given by

$$
\left|\left\{m \in \mathbb{N}_{\lambda, \mu}: m<n\right\}\right| .
$$

Moreover, all these zeros are simple. Hence, the number of simple positive real zeros is nondecreasing and increases to infinity when $n$ tends to infinity. When we only consider a Wronskian of Laguerre polynomials, i.e., $r_{1}=0$ or $r_{2}=0$, then the number of positive real zeros is determined in [37] for almost every $\alpha \in(-1, \infty)$. It is given by the alternating sum of the elements in the partition.

The value of $L_{\lambda, \mu, n}^{(\alpha)}$ at the origin can be computed as in a way similar to the generalized Laguerre polynomial case. It is nonzero when $\alpha>-1$ which is assumed in Lemma 6. Hence, for $n$ large enough, there are $n-|\lambda|-|\mu|$ simple zeros in the orthogonality region $(0, \infty)$ and these zeros are called the regular zeros of $L_{\lambda, \mu, n}^{(\alpha)}$. We denote them by

$$
0<x_{1, n}^{(\alpha)}<x_{2, n}^{(\alpha)}<\cdots<x_{n-|\lambda|-|\mu|, n}^{(\alpha)} .
$$

The remaining $|\lambda|+|\mu|$ zeros are in $\mathbb{C} \backslash[0, \infty)$ and these are the exceptional zeros. We denote them by

$$
z_{1, n}^{(\alpha)}, z_{2, n}^{(\alpha)}, \ldots, z_{|\lambda|+|\mu|, n}^{(\alpha)} .
$$

If the conditions of Lemma 6 are not satisfied, the number of positive real zeros cannot be determined by Sturm-Liouville theory. It is even 
possible that there is a zero at the origin. Therefore, we make the following definition.

Definition 5. For $n \in \mathbb{N}_{\lambda, \mu}$, we use $N(n)$ to denote the number of zeros of $L_{\lambda, \mu, n}^{(\alpha)}$ in $(0, \infty)$ including multiplicity. We call these zeros the regular zeros of $L_{\lambda, \mu, n}^{(\alpha)}$. The remaining $n-N(n)$ zeros are called the exceptional zeros.

We omitted the partitions $\lambda, \mu$ and the parameter $\alpha$ in our notation of $N(n)$ because it should be clear what they are. Obviously, we have that $0 \leq N(n) \leq n$. As stated before, under the conditions of Lemma 6 , the number of regular zeros $N(n)$ is given by (78).

A first result is that we have a lower bound for $N(n)$ and moreover the number of simple regular zeros tends to infinity as the degree $n$ tends to infinity.

THEOREM 2. Let $\alpha+r>-1$. Then, for $n \in \mathbb{N}_{\lambda, \mu}$, we have

$$
n-2(|\lambda|+|\mu|)-r_{2} \leq N(n)
$$

Moreover, the number of simple positive real zeros of $L_{\lambda, \mu, n}^{(\alpha)}$ increases to infinity as $n$ tends to infinity.

Now, we are able to state our asymptotic results. These results justify the conjecture [26, Conjecture 1.1] for exceptional Laguerre polynomials. We prove that the regular zeros of the exceptional Laguerre polynomial have the same asymptotic behavior as the zeros of their classical counterpart and the exceptional zeros converge to the (simple) zeros of the generalized Laguerre polynomial.

\subsection{Mehler-Heine asymptotics}

For Laguerre polynomials, we have the Mehler-Heine asymptotics for all $\alpha \in \mathbb{R}$, see Theorem 6 . This result can be generalized to exceptional Laguerre polynomials. We use $J_{v}$ to denote the Bessel function of the first kind of order $v \in \mathbb{R}[38]$.

THEOREM 3. Take $\alpha \in \mathbb{R}$, then one has

$$
\lim _{n \rightarrow \infty} \frac{(-1)^{r}}{n^{\alpha+r}} L_{\lambda, \mu, n}^{(\alpha)}\left(\frac{x}{4 n}\right)=\Omega_{\lambda, \mu}^{(\alpha)}(0) 2^{\alpha+r} x^{-\frac{\alpha+r}{2}} J_{\alpha+r}(\sqrt{x})
$$

uniformly for $x$ in compact subsets of the complex plane.

The function $x^{-\frac{\alpha+r}{2}} J_{\alpha+r}(\sqrt{x})$ is an entire function in the complex plane with an infinite number of zeros on the positive real line in case $\alpha+r$ $>-1$, and no other zeros. All zeros are simple. Therefore, if we apply Hurwitz theorem [1, Theorem 1.91.3], we obtain the following convergence property for the regular zeros. 
Corollary 1. Assume $\alpha+r>-1$. For a positive integer $k$ and $n \in$ $\mathbb{N}_{\lambda, \mu}$ large enough, let $x_{k, n}^{(\alpha)}$ denote the $k$ th positive real zero of $L_{\lambda, \mu, n}^{(\alpha)}$, see also Theorem 2. If $\Omega_{\lambda, \mu}^{(\alpha)}(0) \neq 0$, then we have

$$
\lim _{n \rightarrow \infty} \sqrt{4 n x_{k, n}^{(\alpha)}}=j_{\alpha+r, k}
$$

where $j_{\alpha+r, k}$ is the kth positive zero of the Bessel function $J_{\alpha+r}$.

\subsection{Weak macroscopic limit of the regular zeros}

Whenever $\alpha+r>-1$, the number of regular zeros $N(n)$ tends to infinity as $n$ tends to infinity by Theorem 2 . The weak scaling limit of the zero-counting measure of the regular zeros is the Marchenko-Pastur distribution $\frac{1}{2 \pi} \sqrt{\frac{4-x}{x}} d x$. This is a generalization of its classical counterpart, see Theorem 7 .

THEOREM 4. Take $\alpha \in \mathbb{R}$ such that $\alpha+r>-1$. Let $0<x_{1, n}^{(\alpha)} \leq \cdots \leq$ $x_{N(n), n}^{(\alpha)}$ denote the regular zeros of the exceptional Laguerre polynomial $L_{\lambda, \mu, n}^{(\alpha)}$ where $n \in \mathbb{N}_{\lambda, \mu}$. Then, for every bounded continuous function $f$ on the positive real line,

$$
\lim _{n \rightarrow \infty} \frac{1}{N(n)} \sum_{j=1}^{N(n)} f\left(\frac{x_{j, n}^{(\alpha)}}{N(n)}\right)=\frac{1}{2 \pi} \int_{0}^{4} f(x) \sqrt{\frac{4-x}{x}} d x .
$$

\subsection{Convergence of the exceptional zeros}

If $\alpha+r>-1$, then the exceptional zeros are attracted by simple zeros of the generalized Laguerre polynomials. We use $z_{1}, \ldots, z_{|\lambda|+|\mu|}$ to denote the zeros of the generalized Laguerre polynomial $\Omega_{\lambda, \mu}^{(\alpha)}$.

THEOREM 5. Take $\alpha \in \mathbb{R}$ such that $\alpha+r>-1$. Let $z_{j}$ be a simple zero of the generalized Laguerre polynomial $\Omega_{\lambda, \mu}^{(\alpha)}$ where $z_{j} \in \mathbb{C} \backslash[0, \infty)$. Then, this zero $z_{j}$ attracts an exceptional zero of the exceptional Laguerre polynomial $L_{\lambda, \mu, n}^{(\alpha)}$ as $n$ tends to infinity at a rate $O\left(n^{-1 / 2}\right)$. That is, for $n$ large enough, we have

$$
\min _{k=1, \ldots, n-N(n)}\left|z_{j}-z_{k, n}^{(\alpha)}\right|<\frac{c}{\sqrt{n}}
$$

for some positive constant $c$ and where $z_{1, n}^{(\alpha)}, \ldots, z_{n-N(n), n}^{(\alpha)}$ denote the exceptional zeros of the exceptional Laguerre polynomial $L_{\lambda, \mu, n}^{(\alpha)}$ with $n \in \mathbb{N}_{\lambda, \mu}$. 


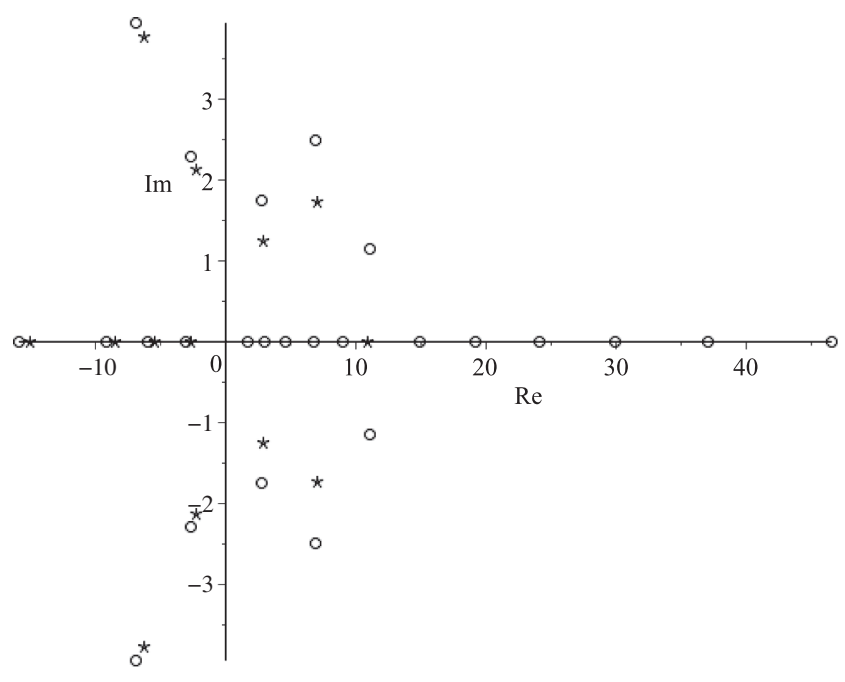

Figure 2. Zeros of the generalized (stars) and exceptional (open circles) Laguerre polynomial associated with $\lambda=(3,2), \mu=(4,2,2), \alpha=1$, and $n=25$.

In the situation of Lemma 6, we have that exceptional Laguerre polynomials form a complete set of orthogonal polynomials. Then, we know that $N(n)=n-|\lambda|-|\mu|$, if $n$ is large enough, and that the zeros of the generalized Laguerre polynomial $\Omega_{\lambda, \mu}^{(\alpha)}$ are all in $\mathbb{C} \backslash[0, \infty)$. If each of these $|\lambda|+|\mu|$ zeros is simple, it follows from Theorem 5 that, for large $n$, each zero $z_{j}$ attracts exactly one exceptional zero of $L_{\lambda, \mu, n}^{(\alpha)}$. Hence, we can relabel the zeros of the exceptional Laguerre polynomial in such a way that $z_{j, n}^{(\alpha)}$ is close to $z_{j}$ and

$$
z_{j, n}^{(\alpha)}=z_{j}+O\left(\frac{1}{\sqrt{n}}\right) \quad \text { as } n \rightarrow \infty .
$$

Our results are numerically verified. In Fig. 2, we plotted the zeros where we set $\alpha=1, \lambda=(3,2)$, and $\mu=(4,2,2)$. The 13 zeros of the generalized Laguerre polynomial, which are indicated by a star, are all simple. The open circles are the zeros of the corresponding exceptional Laguerre polynomial of degree 25. Note that there is one zero of the generalized Laguerre polynomial on the positive real line. Hence, the conditions of Theorem 5 are not satisfied. Nevertheless, it seems that this positive real zero attracts two exceptional zeros.

\subsection{Conjecture of simple zeros}

We do not have a proof that the zeros of $\Omega_{\lambda, \mu}^{(\alpha)}$ are simple, but we offer this as a conjecture, based on numerical evidence. 
Conjecture 1. Take $\alpha>-1$ and let $\lambda$ be an even partition. Then the zeros of the generalized Laguerre polynomial $\Omega_{\lambda, \mu}^{(\alpha)}$ are simple.

In Theorem 5, we stated that the simple zeros of the generalized Laguerre polynomial attract an exceptional zero of the exceptional Laguerre polynomial. This result is comparable to the Hermite case [26, Theorem 2.3]. For the Hermite case, it was conjectured by Veselov [30, Section 6] that all zeros are simple. More concretely, he conjectured that the zeros of a Wronskian of Hermite polynomials are all simple, except possibly at the origin. As already said, we expect a similar conjecture to be true in our case, see Conjecture 1. However, an important difference with the Hermite case is that we need a condition for $\lambda, \mu$, and $\alpha$ to be satisfied. We require a condition because of the following examples:

$$
\begin{array}{ll}
\Omega_{(3,1), \emptyset}^{(5)}(x)=\frac{1}{8}(x-6)^{3}(x-14), & \Omega_{(2,2), \emptyset}^{(-2)}(x)=\frac{1}{12} x^{4}, \\
\Omega_{(1),(2)}^{\left(-\frac{7}{4}\right)}(x)=-\frac{1}{2}\left(x+\frac{3}{4}\right)^{3}, & \Omega_{(3),(3)}^{\left(-\frac{13}{4}\right)}(x)=-\frac{1}{36}\left(x^{2}+\frac{15}{16}\right)^{3} .
\end{array}
$$

It is clear that each of these generalized Laguerre polynomials has a nonsimple zero, either real or nonreal. Numerical simulations seem to suggest that every time that the exceptional Laguerre polynomials form a complete orthogonal system, the corresponding generalized Laguerre polynomial has simple zeros. This is the case when the conditions in Conjecture 1 are satisfied. Moreover, the conjecture holds true for type II exceptional Laguerre polynomials, see [28, Proposition 4.3].

\section{Zeros of exceptional Laguerre polynomials: proofs}

In this section, we give the proofs of the new results which were stated in the previous section.

\subsection{Proof of the lower bound of the regular zeros}

In this section, we prove Theorem 2. The proof is based on the following lemma.

Lemma 10. Let $n \in \mathbb{N}_{\lambda, \mu}$ and take $\alpha \in \mathbb{R}$. Then the exceptional Laguerre polynomial $L_{\lambda, \mu, n}^{(\alpha)}$ is a linear combination of the Laguerre polynomials $L_{n}^{(\alpha+r)}, L_{n-1}^{(\alpha+r)}, \ldots, L_{n-t}^{(\alpha+r)}$ where

$$
t=2(|\lambda|+|\mu|)+r_{2}
$$

is independent of $n$. 
Proof. Set $s=n-|\lambda|-|\mu|+r_{1}$ and consider $L_{\lambda, \mu, n}^{(\alpha)}$ which is defined in (17). By expanding the Wronskian determinant along the last column, one obtains

$$
L_{\lambda, \mu, n}^{(\alpha)}(x)=\sum_{j=0}^{r} Q_{j}(x) \frac{d^{j}}{d x^{j}} L_{s}^{(\alpha)}(x),
$$

where $Q_{j}$ is a polynomial such that

$$
\operatorname{deg} Q_{j} \leq|\lambda|+|\mu|-r_{1}+\min \left\{j, r_{1}\right\}
$$

which is independent of $n$. This upper bound for the degree follows from Proposition 2. Note that $Q_{0}=(-1)^{r_{2}} \Omega_{\tilde{\lambda}, \mu}^{(\alpha+1)}$ where $\tilde{\lambda}_{i}=\lambda_{i}-1$ for $i=1, \ldots, r_{1}$ and $Q_{r}=\Omega_{\lambda, \mu}^{(\alpha)}$. For both polynomials, the upper bound of the degree is attained because of Lemma 1.

The derivative of a Laguerre polynomial is again a Laguerre polynomial of lower degree, but with a shifted parameter, i.e.,

$$
\frac{d^{j}}{d x^{j}} L_{s}^{(\alpha)}(x)=(-1)^{j} L_{s-j}^{(\alpha+j)}(x),
$$

see, e.g. [1, Formula (5.1.14)]. Thus, (83) becomes

$$
L_{\lambda, \mu, n}^{(\alpha)}(x)=\sum_{j=0}^{r} Q_{j}(x)(-1)^{j} L_{s-j}^{(\alpha+j)}(x) .
$$

It is possible to express the Laguerre polynomial as a sum of other Laguerre polynomials with shifted parameters,

$$
L_{n}^{(\alpha)}(x)=\sum_{k=0}^{l}(-1)^{k}\left(\begin{array}{l}
l \\
k
\end{array}\right) L_{n-k}^{(\alpha+l)}(x),
$$

which holds for every positive integer $l$ and $\alpha \in \mathbb{R}$, see [1, Formula (5.1.13)]. Using this in (86) with $n=s-j$ and $l=r-j$, we obtain

$$
\begin{aligned}
L_{\lambda, \mu, n}^{(\alpha)}(x) & =\sum_{j=0}^{r} Q_{j}(x)(-1)^{j} \sum_{k=0}^{r-j}(-1)^{k}\left(\begin{array}{c}
s-j \\
k
\end{array}\right) L_{s-j-k}^{(\alpha+r)}(x) \\
& =\sum_{j=0}^{r} \tilde{Q}_{j}(x) L_{s-j}^{(\alpha+r)}(x),
\end{aligned}
$$

where $\tilde{Q}_{j}$ is a certain polynomial of degree

$$
\operatorname{deg} \tilde{Q}_{j} \leq|\lambda|+|\mu|-r_{1}+\min \left\{j, r_{1}\right\}
$$

Next, we use the three-term recurrence satisfied by the Laguerre polynomials from which it follows that $x L_{k}^{(\alpha+r)}$ is a linear combination of the 
polynomials of degrees $k+1, k$, and $k-1$. Using the recurrence repeatedly, we see that $\tilde{Q}_{j} L_{s-j}^{(\alpha+r)}$ is a linear combination of Laguerre polynomials $L_{k}^{(\alpha+r)}$ with $k$ in the range

$$
s-j-\operatorname{deg} \tilde{Q}_{j} \leq k \leq s-j+\operatorname{deg} \tilde{Q}_{j} .
$$

For $j=0, \ldots, r$ we have by the definitions of $s$ and $t$ and the degree bound (88) that

$$
s-j-\operatorname{deg} \tilde{Q}_{j} \geq s-r-(|\lambda|+|\mu|)=n-t
$$

and

$$
s-j+\operatorname{deg} \tilde{Q}_{j} \leq s+(|\lambda|+|\mu|)-r_{1}=n .
$$

Thus, for each $j=0, \ldots, r$, we have that $\tilde{Q}_{j} L_{s-j}^{(\alpha+r)}$ is a linear combination of $L_{k}^{(\alpha+r)}$ with $n-t \leq k \leq n$ and the lemma follows because of (87).

Remark 7. In [26], there is an analogous result for exceptional Hermite polynomials, see Lemma 4.1. However, the statement and proof of this result in [26] contain a mistake. The exceptional Hermite polynomial of degree $n$ associated with a partition $\lambda$ is a linear combination of the Hermite polynomials $H_{n}, \ldots, H_{n-t}$ where $t=2|\lambda|$, while it is stated in [26] that $t=|\lambda|+r$, where $r$ is the length of $\lambda$. This mistake, however, does not affect the further results in [26].

With Lemma 10, we can prove Theorem 2.

Proof of Theorem 2. From Lemma 10 and the orthogonality of the polynomials $L_{k}^{(\alpha+r)}$ on $[0, \infty)$, which holds because $\alpha+r>-1$, we obtain

$$
\int_{0}^{\infty} Q(x) L_{\lambda, \mu, n}^{(\alpha)}(x) x^{\alpha+r} e^{-x} d x=0
$$

whenever $Q$ is a polynomial of degree $<n-t$ where the number $t$ is as in Lemma 10. This forces $L_{\lambda, \mu, n}^{(\alpha)}$ to have at least $n-t$ zeros in $(0, \infty)$ with odd multiplicity. Otherwise, we can construct a polynomial of degree $<n-t$ in such a way that $Q L_{\lambda, \mu, n}^{(\alpha)}$ does not change sign in $(0, \infty)$ and this would contradict (89). Thus,

$$
N(n) \geq n-t=n-2(|\lambda|+|\mu|)-r_{2} .
$$

Moreover, the number of zeros with odd multiplicity is bounded by this number $n-t$. The number of zeros with multiplicity at least 3 is trivially bounded by $\frac{n}{3}$. Hence, the number of simple regular zeros is at least $\frac{2 n}{3}-t$ and therefore tends to infinity as $n$ tends to infinity.

There is another consequence of Lemma 10 that we state here for future reference. 
COROLlaRY 2. Take $\alpha \in \mathbb{R}$ such that $\alpha+r>-1$. Let $n \in \mathbb{N}_{\lambda, \mu}$ such that $n>2(|\lambda|+|\mu|)+r_{2}$. Let $0<a_{1, n}^{(\alpha+r)}<a_{2, n}^{(\alpha+r)}<\cdots<a_{n, n}^{(\alpha+r)}$ denote the real and simple zeros of the Laguerre polynomial $L_{n}^{(\alpha+r)}$. Then, at least $n-2(|\lambda|+|\mu|)-r_{2}$ intervals $\left(a_{k, n}^{(\alpha+r)}, a_{k+1, n}^{(\alpha+r)}\right)$, where $1 \leq k<n$, contain a zero of the exceptional Laguerre polynomial $L_{\lambda, \mu, n}^{(\alpha)}$.

Proof. This follows from Lemma 10 as was shown by Beardon and Driver [39, Theorem 3.2] for arbitrary orthogonal polynomials on the real line.

The lower bound in Theorem 2 also follows immediately from Corollary 2.

\subsection{Proof of Mehler-Heine asymptotics}

Mehler-Heine asymptotics describe the behavior of orthogonal polynomials near the edges of their support. For the Laguerre polynomials, this reads as follows [1, Theorem 8.1.3].

THEOREM 6. Take $\alpha \in \mathbb{R}$, then one has

$$
\lim _{n \rightarrow \infty} \frac{1}{n^{\alpha}} L_{n}^{(\alpha)}\left(\frac{x}{4 n}\right)=2^{\alpha} x^{-\frac{\alpha}{2}} J_{\alpha}(\sqrt{x}),
$$

uniformly for $x$ in compact subsets of the complex plane.

According to Theorem 3, a similar asymptotic behavior holds for the exceptional Laguerre polynomials as we are now going to prove.

Proof of Theorem 3. Set $s=n-|\lambda|-|\mu|+r_{1}$ and write $L_{\lambda, \mu, n}^{(\alpha)}(x)$ as in (83). Clearly, if we do the expansion of (17) along the last column, we find $Q_{r}=\Omega_{\lambda, \mu}^{(\alpha)}$, and therefore

$$
L_{\lambda, \mu, n}^{(\alpha)}(x)=\sum_{j=0}^{r-1} Q_{j}(x) \frac{d^{j}}{d x^{j}} L_{s}^{(\alpha)}(x)+\Omega_{\lambda, \mu}^{(\alpha)}(x) \frac{d^{r}}{d x^{r}} L_{s}^{(\alpha)}(x),
$$

where $Q_{j}$ is a polynomial of degree at most $|\lambda|+|\mu|-r_{1}+\min \left\{j, r_{1}\right\}$ which does not depend on $n$.

The limit (90) also holds if we replace $L_{n}^{(\alpha)}$ by $L_{s}^{(\alpha)}$, where $s=n-c$ for some constant $c$. Thus,

$$
\lim _{n \rightarrow \infty} \frac{1}{n^{\alpha}} L_{s}^{(\alpha)}\left(\frac{x}{4 n}\right)=2^{\alpha} x^{-\frac{\alpha}{2}} J_{\alpha}(\sqrt{x})
$$

uniformly for $x$ in compact subsets of the complex plane. Because of the uniform convergence, (92) can be differentiated with respect to $x$ any 
number of times. Hence, for every nonnegative integer $j$,

$$
\lim _{n \rightarrow \infty} \frac{1}{4^{j} n^{\alpha+j}}\left(\frac{d^{j}}{d x^{j}} L_{s}^{(\alpha)}\right)\left(\frac{x}{4 n}\right)=\frac{d^{j}}{d x^{j}}\left(2^{\alpha} x^{-\frac{\alpha}{2}} J_{\alpha}(\sqrt{x})\right) .
$$

In particular,

$$
\left(\frac{d^{j}}{d x^{j}} L_{s}^{(\alpha)}\right)\left(\frac{x}{4 n}\right)=O\left(n^{\alpha+j}\right)
$$

as $n \rightarrow \infty$ uniformly for $x$ in compacts. In fact, the previous equality can also be obtained directly because the derivative of the Laguerre polynomials is again a Laguerre polynomial (85) and therefore we can apply (90) with the correct parameter.

Hence, the limiting behavior of

$$
\frac{1}{n^{\alpha+r}} L_{\lambda, \mu, n}^{(\alpha)}\left(\frac{x}{4 n}\right)
$$

is determined by the last term in (91) only. We find from (91) and (93) with $j=r$,

$$
\lim _{n \rightarrow \infty} \frac{1}{n^{\alpha+r}} L_{\lambda, \mu, n}^{(\alpha)}\left(\frac{x}{4 n}\right)=\Omega_{\lambda, \mu}^{(\alpha)}(0) 4^{r} \frac{d^{r}}{d x^{r}}\left(2^{\alpha} x^{-\frac{\alpha}{2}} J_{\alpha}(\sqrt{x})\right) .
$$

Because of the identity for Bessel functions

$$
\frac{d}{d x}\left(x^{-\frac{\alpha}{2}} J_{\alpha}(\sqrt{x})\right)=-\frac{1}{2} x^{-\frac{\alpha+1}{2}} J_{\alpha+1}(\sqrt{x})
$$

we obtain the desired result (80).

Next, we apply Hurwitz's theorem to obtain the asymptotic convergence for the regular zeros as stated in Corollary 1 . We need the assumption that $\Omega_{\lambda, \mu}^{(\alpha)}(0) \neq 0$ so that the right-hand side of (80) is not identically zero.

Proof of Corollary 1. Applying Hurwitz's theorem to (80) gives us that those zeros of $L_{\lambda, \mu, n}^{(\alpha)}\left(\frac{x}{4 n}\right)$ that do not tend to infinity tend to the zeros of $x^{-(\alpha+r) / 2} J_{\alpha+r}(\sqrt{x})$ as $n$ tends to infinity. All these limiting zeros are simple and lie on the positive real line.

Because the zeros are simple, Hurwitz's theorem also says that each zero of $J_{\alpha+r}(\sqrt{x})$ attracts exactly one zero of $L_{\lambda, \mu, n}^{(\alpha)}\left(\frac{x}{4 n}\right)$ as $n \rightarrow \infty$. This zero has to be real for large enough $n$, because its complex conjugate is a zero as well and if it were not real for large $n$, then two zeros of $L_{\lambda, \mu, n}^{(\alpha)}\left(\frac{x}{4 n}\right)$ would approach the same simple zero of $J_{\alpha+r}(\sqrt{x})$.

\subsection{Proof of the weak macroscopic limit of the regular zeros}

In this section, we prove that the limit behavior of the zero-counting measure of the regular zeros of the exceptional Laguerre polynomials is 
given by the Marchenko-Pastur distribution. It is a generalization of the following well-known limit of the zero-counting measure for Laguerre polynomials, see, e.g. [40, Theorem 1].

THEOREM 7. Let $0<a_{1, n}^{(\alpha)}<a_{2, n}^{(\alpha)}<\cdots<a_{n, n}^{(\alpha)}<\infty$ denote the zeros of the Laguerre polynomial $L_{n}^{(\alpha)}$ where $\alpha>-1$. Then, for any bounded continuous function $f:[0, \infty) \rightarrow \mathbb{R}$, it is true that

$$
\lim _{n \rightarrow \infty} \frac{1}{n} \sum_{j=1}^{n} f\left(\frac{a_{j, n}^{(\alpha)}}{n}\right)=\frac{1}{2 \pi} \int_{0}^{4} f(x) \sqrt{\frac{4-x}{x}} d x .
$$

Knowing Theorem 7, we prove Theorem 4 in essentially the same way as Theorem 2.2 in [26], which dealt with the semicircle law for the scaled zeros of exceptional Hermite polynomials.

Proof of Theorem 4. Suppose $\alpha+r>-1$. Take $n \in \mathbb{N}_{\lambda, \mu}$ such that $n>$ $2(|\lambda|+|\mu|)+r_{2}$. Let $0<a_{1, n}^{(\alpha+r)}<a_{2, n}^{(\alpha+r)}<\cdots<a_{n, n}^{(\alpha+r)}$ denote the zeros of the Laguerre polynomial $L_{n}^{(\alpha+r)}$. From Lemma 10 and Corollary 2, it follows that at least $n-2(|\lambda|+|\mu|)-r_{2}$ intervals $\left(a_{j, n}^{(\alpha+r)}, a_{j+1, n}^{(\alpha+r)}\right)$, where $1 \leq j<n$, contain a zero of the exceptional Laguerre polynomial $L_{\lambda, \mu, n}^{(\alpha)}$.

For any choice of $\xi_{j, n} \in\left(a_{j, n}^{(\alpha+r)}, a_{j+1, n}^{(\alpha+r)}\right)$ for every $j=1, \ldots, n-1$, we get that the limit (94) is still satisfied, i.e.,

$$
\lim _{n \rightarrow \infty} \frac{1}{n-1} \sum_{j=1}^{n-1} f\left(\frac{\xi_{j, n}}{n}\right)=\frac{1}{2 \pi} \int_{0}^{4} f(x) \sqrt{\frac{4-x}{x}} d x
$$

For $n$ large, we can take $\xi_{j, n}$ to be equal to a zero of of $L_{\lambda, \mu, n}^{(\alpha)}$ for at least $n-2(|\lambda|+|\mu|)-r_{2}$ values of $j$. By dropping the other indices in the sum, the limit will not be affected as $f$ is bounded. Next, one can extend the sum by including the remaining positive real zeros of $L_{\lambda, \mu, n}^{(\alpha)}$ because their number remains bounded as $n$ increases. Hence, we obtain (81).

\subsection{Proof of the convergence of the exceptional zeros}

In this section, we prove Theorem 5 which deals with the convergence of the exceptional zeros. Recall the weight

$$
W_{\lambda, \mu}^{(\alpha)}(x)=\frac{x^{\alpha+r} e^{-x}}{\left(\Omega_{\lambda, \mu}^{(\alpha)}(x)\right)^{2}}
$$

that appeared in Lemma 6 for $x \in(0, \infty)$. In this section, we view (95) as a meromorphic function in $\mathbb{C} \backslash\{0\}$ with poles at the zeros of $\Omega_{\lambda, \mu}^{(\alpha)}$. We also consider a general parameter $\alpha \in \mathbb{R}$ and partitions $\lambda$ and $\mu$. We will need the following property. 
Lemma 11. Take $\alpha \in \mathbb{R}$. For every $n \in \mathbb{N}_{\lambda, \mu}$, we have that

$$
\left(L_{\lambda, \mu, n}^{(\alpha)}\right)^{2} W_{\lambda, \mu}^{(\alpha)}
$$

has zero residue at each of its poles in $\mathbb{C} \backslash\{0\}$.

Proof. We apply a Darboux-Crum transformation to the differential operator (2) with eigenfunctions $\varphi_{n_{1}}^{(\alpha)}, \ldots, \varphi_{n_{r_{1}}}^{(\alpha)}, \psi_{m_{1}}^{(\alpha)}, \ldots, \psi_{m_{r_{2}}}^{(\alpha)}$, see Table 2 in Section 2.1. It leads to a new differential operator

$$
y \mapsto-y^{\prime \prime}+V_{\lambda, \mu} y
$$

with potential

$$
V_{\lambda, \mu}(x)=x^{2}+\frac{4 \alpha^{2}-1}{4 x^{2}}-2 \frac{d^{2}}{d x^{2}} \log \left(\mathrm{Wr}\left[\varphi_{n_{1}}^{(\alpha)}, \ldots, \varphi_{n_{r_{1}}}^{(\alpha)}, \psi_{m_{1}}^{(\alpha)}, \ldots, \psi_{m_{r_{2}}}^{(\alpha)}\right]\right) \text {. }
$$

The differential operator (96) has eigenfunctions of the form

$$
\frac{\mathrm{Wr}\left[\varphi_{n_{1}}^{(\alpha)}, \ldots, \varphi_{n_{r_{1}}}^{(\alpha)}, \psi_{m_{1}}^{(\alpha)}, \ldots, \psi_{m_{r_{2}}}^{(\alpha)}, \varphi_{s}^{(\alpha)}\right]}{\operatorname{Wr}\left[\varphi_{n_{1}}^{(\alpha)}, \ldots, \varphi_{n_{r_{1}}}^{(\alpha)}, \psi_{m_{1}}^{(\alpha)}, \ldots, \psi_{m_{r_{2}}}^{(\alpha)}\right]}
$$

where $s \geq 0$ and $s \neq n_{j}$ for every $j=1, \ldots, r_{1}$.

Using (12) and (13), we can express the Wronskian in (97) as a Wronskian for the functions $f_{1}, \ldots, f_{r}$ from (8) to (9), and it follows from (7) that

$$
\mathrm{Wr}\left[\varphi_{n_{1}}^{(\alpha)}, \ldots, \varphi_{n_{r_{1}}}^{(\alpha)}, \psi_{m_{1}}^{(\alpha)}, \ldots, \psi_{m_{r_{2}}}^{(\alpha)}\right]=2^{\frac{r(r-1)}{2}} x^{\alpha r+\frac{r^{2}}{2}} e^{-\frac{r}{2} x^{2}} \Omega_{\lambda, \mu}^{(\alpha)}\left(x^{2}\right)
$$

Hence,

$$
V_{\lambda, \mu}(x)=x^{2}+2 r+\frac{4(\alpha+r)^{2}-1}{4 x^{2}}-2 \frac{d^{2}}{d x^{2}} \log \left(\Omega_{\lambda, \mu}^{(\alpha)}\left(x^{2}\right)\right) .
$$

Similarly, the eigenfunction (98) can be written as:

$$
2^{r} x^{\alpha+\frac{1}{2}+r} e^{-\frac{1}{2} x^{2}} \frac{L_{\lambda, \mu, n}^{(\alpha)}\left(x^{2}\right)}{\Omega_{\lambda, \mu}^{(\alpha)}\left(x^{2}\right)}
$$

if we choose $n \in \mathbb{N}_{\lambda, \mu}$ and $s=n-|\lambda|-|\mu|+r_{1}$.

We now use the fact that the operator (96) has trivial monodromy at every point $p \in \mathbb{C} \backslash\{0\}$, see [18, Proposition 5.21]. This means that any eigenfunction of (96) is meromorphic around $p$.

From (99), we see that $p \neq 0$ is a pole of $V_{\lambda, \mu}$ if and only if $p^{2}$ is a zero of $\Omega_{\lambda, \mu}^{(\alpha)}$. If $p^{2}$ is a zero of order $d_{p} \geq 0$, then

$$
V_{\lambda, \mu}(x)=2 d_{p}(x-p)^{-2}+O(1) \quad \text { as } x \rightarrow p .
$$


By Proposition 3.3 of [41], we then have that $2 d_{p}=v_{p}\left(v_{p}+1\right)$ for some positive integer $v_{p}$. Moreover, every eigenfunction $f$ has a Laurent expansion around $p$

$$
f(x)=(x-p)^{-v_{p}} \sum_{j=0}^{\infty} f_{j}(x-p)^{j}
$$

with $f_{2 j-1}=0$ for $j=1, \ldots, v_{p}$. This property implies that $f^{2}$ has zero residue at $x=p$.

This in particular holds for the eigenfunction (100) and thus

$$
x^{2 \alpha+1+2 r} e^{-x^{2}}\left(\frac{L_{\lambda, \mu, n}^{(\alpha)}\left(x^{2}\right)}{\Omega_{\lambda, \mu}^{(\alpha)}\left(x^{2}\right)}\right)^{2}=x\left(L_{\lambda, \mu, n}^{(\alpha)}\left(x^{2}\right)\right)^{2} W_{\lambda, \mu}^{(\alpha)}\left(x^{2}\right)
$$

has zero residue at each of its poles in $\mathbb{C} \backslash\{0\}$. Now, let $p^{2} \in \mathbb{C} \backslash\{0\}$ be a pole of $\left(L_{\lambda, \mu, n}^{(\alpha)}\right)^{2} W_{\lambda, \mu}^{(\alpha)}$. The residue is given by

$$
\frac{1}{2 \pi i} \oint_{\gamma_{p^{2}}}\left(L_{\lambda, \mu, n}^{(\alpha)}(z)\right)^{2} W_{\lambda, \mu}^{(\alpha)}(z) d z
$$

where $\gamma_{p^{2}}$ is a small circle going around $p^{2}$ in counterclockwise direction. By a change of variables $z \mapsto z^{2}$, we obtain that the residue is equal to

$$
\frac{1}{\pi i} \oint_{\gamma_{p}} z\left(L_{\lambda, \mu, n}^{(\alpha)}\left(z^{2}\right)\right)^{2} W_{\lambda, \mu}^{(\alpha)}\left(z^{2}\right) d z
$$

with $\gamma_{p}$ around $p$, and $p$ is a pole of (101). This integral is zero, because (101) has zero residues, and we see that (102) is zero. The lemma follows.

Note that if we replace $\varphi_{s}^{(\alpha)}$ by $\psi_{s}^{(\alpha)}$ in (98), we obtain other eigenfunctions of the differential operator (96). These eigenfunctions can be written in terms of $\tilde{L}_{\lambda, \mu, n}^{(\alpha)}\left(x^{2}\right)$ and $\Omega_{\lambda, \mu}^{(\alpha)}\left(x^{2}\right)$. Now, we are able to prove the asymptotic behavior of the exceptional zeros.

Proof of Theorem 5. Let $z_{j}$ be simple zero of $\Omega_{\lambda, \mu}^{(\alpha)}$ where $z_{j} \in \mathbb{C} \backslash$ $[0, \infty)$. Then, $z_{j}$ is a double pole of $W_{\lambda, \mu}^{(\alpha)}$ and by (11)

$$
\left(x-z_{j}\right)^{2}\left(L_{\lambda, \mu, n}^{(\alpha)}(x)\right)^{2} W_{\lambda, \mu}^{(\alpha)}(x)=C_{0}+C_{1}\left(x-z_{j}\right)+O\left(\left(x-z_{j}\right)^{2}\right) \quad \text { as } x \rightarrow z_{j}
$$

for a certain constant $C_{0}$ and $C_{1}=0$. We may assume $C_{0} \neq 0$, otherwise $z_{j}$ is a zero of $L_{\lambda, \mu, n}^{(\alpha)}$ as well and then (82) is clearly satisfied.

Because $C_{0} \neq 0$, we can take an analytic logarithm of (103) in the neighborhood of $z_{j}$, and because $C_{1}=0$, its derivative vanishes at $z_{j}$. The 
logarithmic derivative of the left-hand side of (103) is

$$
\frac{2}{x-z_{j}}+2 \sum_{k=1}^{N(n)} \frac{1}{x-x_{k, n}^{(\alpha)}}+2 \sum_{k=1}^{n-N(n)} \frac{1}{x-z_{k, n}^{(\alpha)}}+\frac{\alpha+r}{x}-1-2 \sum_{k=1}^{|\lambda|+|\mu|} \frac{1}{x-z_{k}}
$$

where, as before, $0<x_{1, n}^{(\alpha)} \leq \cdots \leq x_{N(n), n}^{(\alpha)}$ are the regular zeros and $z_{1, n}^{(\alpha)}$, $\ldots, z_{n-N(n), n}^{(\alpha)}$ are the exceptional zeros of the exceptional Laguerre polynomial and $z_{1}, \ldots, z_{|\lambda|+|\mu|}$ are the zeros of $\Omega_{\lambda, \mu}^{(\alpha)}$. Thus, (104) vanishes because $x \rightarrow z_{j}$, and it gives us the identity

$$
\sum_{k=1}^{N(n)} \frac{1}{z_{j}-x_{k, n}^{(\alpha)}}+\sum_{k=1}^{n-N(n)} \frac{1}{z_{j}-z_{k, n}^{(\alpha)}}=\frac{1}{2}-\frac{\alpha+r}{2 z_{j}}+\sum_{\substack{k=1 \\ k \neq j}}^{|\lambda|+|\mu|} \frac{1}{z_{j}-z_{k}} .
$$

On the interval $[0,1]$, the number of zeros of $L_{n}^{(\alpha+r)}$ grows roughly like $c \sqrt{n}$ as $n$ tends to infinity for some positive constant $c$, see [1, Theorem 6.31.3]. Because of Corollary 2, the same holds true for the number of zeros of $L_{\lambda, \mu, n}^{(\alpha)}$ in $[0,1]$. We now distinguish between $z_{j}$ being real and $z_{j}$ being nonreal.

Case 1: $\operatorname{Im}\left(z_{j}\right) \neq 0$.

As the nonreal roots of polynomials with real coefficients come in conjugate pairs, we may assume that $\operatorname{Im}\left(z_{j}\right)>0$. The imaginary part of the first sum in the left-hand side of (105) simplifies to

$$
\operatorname{Im}\left(\sum_{k=1}^{N(n)} \frac{1}{z_{j}-x_{k, n}^{(\alpha)}}\right)=-\sum_{k=1}^{N(n)} \frac{\operatorname{Im}\left(z_{j}\right)}{\left|z_{j}-x_{k, n}^{(\alpha)}\right|^{2}} .
$$

All terms in this sum have the same sign, namely, the sign of $\operatorname{Im}\left(z_{j}\right)$ which is positive. Therefore, by restricting the sum to the zeros which are in the interval $[0,1]$ and using the fact that there are at least $c \sqrt{n}$ such zeros, we have that for $n$ large enough

$$
\begin{aligned}
\operatorname{Im}\left(\sum_{k=1}^{N(n)} \frac{1}{z_{j}-x_{k, n}^{(\alpha)}}\right) & <-\sum_{k=1}^{\lfloor c \sqrt{n}\rfloor} \frac{\operatorname{Im}\left(z_{j}\right)}{\left|z_{j}-x_{k, n}^{(\alpha)}\right|^{2}} \\
& <-c_{1} \sqrt{n}
\end{aligned}
$$

for some constant $c_{1}>0$. To obtain the second inequality, we used that for $x_{k, n}^{(\alpha)} \in[0,1]$,

$$
\left|z_{j}-x_{k, n}^{(\alpha)}\right|^{2} \leq \max \left\{\left|z_{j}-1\right|^{2},\left|z_{j}\right|^{2}\right\}
$$


where the right-hand side is independent of $n$.

The right-hand side of (105) does not depend on $n$. Therefore, to balance the terms, one has that for $n$ large enough

$$
\operatorname{Im}\left(\sum_{k=1}^{n-N(n)} \frac{1}{z_{j}-z_{k, n}^{(\alpha)}}\right)>c_{1} \sqrt{n} .
$$

This is a finite sum and the number of terms is bounded by $2(|\lambda|+|\mu|)-r_{2}$ because of Theorem 2. Thus, there is at least one term that is also of order $\sqrt{n}$. Hence, there is a constant $c_{2}>0$ such that for every large enough $n$ there exists a zero $z_{k, n}^{(\alpha)}$ with

$$
\operatorname{Im}\left(\frac{1}{z_{j}-z_{k, n}^{(\alpha)}}\right)>c_{2} \sqrt{n} .
$$

The fact that $\operatorname{Im}\left(\frac{1}{z}\right)<\frac{1}{|z|}$ implies that from (107), we find

$$
\left|z_{j}-z_{k, n}^{(\alpha)}\right|<\frac{1}{c_{2} \sqrt{n}},
$$

which ends the proof in this case.

Case 2: $\operatorname{Im}\left(z_{j}\right)=0$.

Because $z_{j} \notin[0, \infty)$, by assumption, we then have that $z_{j}$ is an element of the negative real axis. We can give a similar argument as in case 1; however, one has to consider the real part of (105) and (106) needs to be replaced by

$$
z_{j}-x_{l, n}^{(\alpha)} \geq z_{j}-1
$$

The two cases complete the proof of inequality (82) and the theorem follows.

\section{Acknowledgments}

Arno Kuijlaars is supported by long-term structural funding-Methusalem grant of the Flemish Government, the Belgian Interuniversity Attraction Pole P07/18, KU Leuven Research Grant OT/12/073, and FWO Flanders projects G.0934.13 and G.0864.16.

\section{References}

1. G. SzegÖ, Orthogonal Polynomials, 4th ed., American Mathematical Society, Providence, Rhode Island, 1975. 
2. A. J. DURÁn, Exceptional Meixner and Laguerre orthogonal polynomials, J. Approx. Theory 184:176-208 (2014).

3. D. Gómez-Ullate, N. Kamran, and R. Milson, An extended class of orthogonal polynomials defined by a Sturm-Liouville problem, J. Math. Anal. Appl. 359:352-367 (2009).

4. D. Gómez-Ullate, N. Kamran, and R. Milson, An extension of Bochners problem: Exceptional invariant subspaces, J. Approx. Theory 162:987-1006 (2010).

5. D. Gómez-Ullate, N. Kamran, and R. Milson, Two-step Darboux transformations and exceptional Laguerre polynomials, J. Math. Anal. Appl. 387:410-418 (2012).

6. C. L. Ho, S. Odake, and R. Sasaki, Properties of the exceptional $\left(X_{l}\right)$ Laguerre and Jacobi polynomials, Symmetry Integr. Geom. 7:107 (2011).

7. C. Liaw, L. L. Littlejohn, R. Milson, and J. Stewart, The spectral analysis of three families of exceptional Laguerre polynomials, J. Approx. Theory 202:5-41 (2016).

8. S. OdAKe and R. SASAKI, Another set of infinitely many exceptional $\left(X_{l}\right)$ Laguerre polynomials, Phys. Lett. B 684:173-176 (2010).

9. S. ODAKE and R. SASAKI, Infinitely many shape invariant potentials and new orthogonal polynomials, Phys. Lett. B 679:414-417 (2009).

10. R. Sasaki, S. Tsujimoto, and A. Zhedanov, Exceptional Laguerre and Jacobi polynomials and the corresponding potentials through Darboux-Crum transformations, Jo. Phys. A: Math. Theor. 43:315204 (2010).

11. Y. Grandati and C. Quesne, Disconjugacy, regularity of multi-indexed rationally extended potentials, and Laguerre exceptional polynomials, J. Math. Phys. 54:073512 (2013).

12. S. Odake, Equivalences of the multi-indexed orthogonal polynomials, J. Math. Phys. 55:013502 (2014).

13. S. ODAKE, New determinant expressions of the multi-indexed orthogonal polynomials in discrete quantum mechanics, Progr. Theor. Exp. Phys. 053A01:36 (2017).

14. S. ODAKE and R. SASAKI, Exactly solvable quantum mechanics and infinite families of multi-indexed orthogonal polynomials, Phys. Lett. B 702:164-170 (2011).

15. S. OdAKe and R. SASAKI, Simplified expressions of the multi-indexed Laguerre and Jacobi polynomials, SIGMA 13:10 (2017).

16. R. SASAKI and K. TAKEMURA, Global solutions of certain second-order differential equations with a high degree of apparent singularity, SIGMA 8:085 (2012).

17. A. J. DurÁN and M. PÉREZ, Admissibility condition for exceptional Laguerre polynomials, J. Math. Anal. Appl. 424:1042-1053 (2015).

18. M. García-Ferrero, D. Gómez-Ullate, and R. Milson, A Bochner type classification theorem for exceptional orthogonal polynomials, preprint arXiv:1603.04358.

19. M. M. Crum, Associated Sturm-Liouville systems, Quart. J. Math. 6:121-127 (1955).

20. G. Darboux, Sur une proposition relative aux équations linéaires, Comptes Rendus Acad. Sci. 94:1456-1459 (1882).

21. C. L. Ho, Dirac(-Pauli), Fokker-Planck equations and exceptional Laguerre polynomials, Ann. Phys. 326:797-807 (2011).

22. C. QUeSNe, Exceptional orthogonal polynomials and new exactly solvable potentials in quantum mechanics, J. Phys. Conf. Ser. 380:012016 (2012).

23. I. Marquette and C. Quesne, New families of superintegrable systems from Hermite and Laguerre exceptional orthogonal polynomials, J. Math. Phys. 54:042102 (2013).

24. G. E. Andrews, The Theory of Partitions, Cambridge University Press, New York, 1998. 
25. D. Gómez-Ullate, Y. Grandati, and R. Milson, Rational extensions of the quantum harmonic oscillator and exceptional Hermite polynomials, J. Phys. A: Math. Theor. 47:015203 (2014).

26. A. B. J. Kuillaars and R. Milson, Zeros of exceptional Hermite polynomials, $J$. Approx. Theory 200:28-39 (2015).

27. C. L. Ho and R. SASAKI, Zeros of the exceptional Laguerre and Jacobi polynomials, Int. Sch. Res. Notices 2012:27 (2012).

28. D. Gómez-Ullate, F. Marcellán, and R. Milson, Asymptotic and interlacing properties of zeros of exceptional Jacobi and Laguerre polynomials, J. Math. Anal. Appl. 399:480-495 (2013).

29. Á.P. Hovráth, The electrostatic properties of zeros of exceptional Laguerre and Jacobi polynomials and stable interpolation, J. Approx. Theory 194:87-107 (2015).

30. G. Felder, A. D. Hemery, and A. P. Veselov, Zeros of Wronskians of Hermite polynomials and Young diagrams, Physica D 241:2131-2137 (2012).

31. D. Gómez-Ullate, Y. Grandati, and R. Milson, Durfee rectangles and pseudoWronskian equivalences for Hermite polynomials, preprint arXiv:1612.05514.

32. G. P. CURBERA and A. J. DURÁN, Invariant properties for Wronskian type determinants of classical and classical discrete orthogonal polynomials under an involution of sets of positive integers, preprint arXiv:1612.07530.

33. A. J. DurÁn and M. D. DE LA IgLeSIA, Constructing bispectral orthogonal polynomials from the classical discrete families of Charlier, Meixner and Krawtchouk, Constr. Approx. 41:49-91 (2015).

34. K. Takemura, Multi-indexed Jacobi polynomials and Maya diagrams, J. Math. Phys. 55:113501 (2014).

35. J. Olsson, Combinatorics and Representations of Finite Groups, Fachbereich Mathematik, Universität Essen, Germany, 1993.

36. B. Simon, Sturm oscillation and comparison theorems, in Sturm-Liouville Theory, Past and Present (W. O. Amrein, A. M. Hinz, and D. B. Pearson, Eds.), pp. 29-43, Birkhäuser Verlag, Basel, 2005.

37. M. García-Ferrero and D. Gómez-Ullate, Oscillation theorems for the Wronskian of an arbitrary sequence of eigenfunctions of Schrödingerâs equation, Lett. Math. Phys. 105:551-573 (2015).

38. G. Watson, A Treatise on the Theory of Bessel Functions, Cambridge University Press, New York, 1944.

39. A. F. BEARDon and K. A. Driver, The zeros of linear combinations of orthogonal polynomials, J. Approx. Theory 137:179-186 (2005).

40. W. Gawronski, On the asymptotic distribution of the zeros of Hermite, Laguerre, and Jonquière polynomials, J. Approx. Theory 50:214-231 (1987).

41. J. J. Duistermant and F. A. GrünBAum, Differential equations in the spectral parameter, Commun. Math. Phys. 103:177-240 (1986).

\section{Katholieke Universiteit LeUVEN}

(Received August 9, 2017) 